\title{
Hyperdoctrines and the Ontology of Stratified Semantics
}

\author{
Shay Allen Logan
}

\begin{abstract}
I present a version of Kit Fine's stratified semantics for the logic RWQ and define a natural family of related structures called RW hyperdoctrines. After proving that RWQ is sound with respect to RW hyperdoctrines, we show how to construct, for each stratified model, a hyperdoctrine that verifies precisely the same sentences. Completeness of RWQ for hyperdoctrinal semantics then follows from completeness for stratified semantics, which is proved in an appendix. By examining the base category of RW hyperdoctrines, we find reason to be worried about the ontology of stratified models.
\end{abstract}

\section{Introduction}

Hyperdoctrines, introduced in [14], provide algebraic semantics for a broad range of formal systems. Saying anything more than this is, unfortunately, a bit difficult since (as pointed out in [20]) 'there is no standard terminology for the various kinds of hyperdoctrine which have been used in categorical logic.' This is an admittedly unfortunate situation.

Nonetheless, there are some commonalities among the various features on offer. Of interest to the project pursued here is that hyperdoctrines of all stripes involve a base category $\mathbf{C}$ and assignments of information to the objects and arrows of $\mathbf{C}$. ${ }^{1}$ Further, the interpretation of the base category $\mathbf{C}$ also remains consistent: its objects are types and its arrows are terms. ${ }^{2}$ So there is a natural sense in which hyperdoctrines carry ontological information. More concretely, presenting the semantics of a given theory th as a hyperdoctrine with base category $\mathbf{C}$ gives us reason (it would

Shay Allen Logan

Kansas State University, Manhattan, KS, USA, e-mail: shay.a.logan@gmail.com

${ }^{1}$ For preliminaries on category theory, see e.g. [13].

2 This can be fleshed out more, but it would take us too far afield to do so - the interested reader is referred to [24] for details. 
seem) to think that $t h$ presupposes there is a different sort of object corresponding to every object in $\mathbf{C}$ and that there are (perhaps merely possible) objects corresponding to every arrow in $\mathbf{C}$.

A seemingly quite different sort of structure - Kit Fine's stratified models; see [9] - gives the standard semantics for quantified relevance logics. Fine interprets stratified semantics as having an ontology consisting of arbitrary objects. And it is in fact the case that, once one becomes familiar with the ins and outs of stratified models, one can squint at the objects lurking in their domains and see arbitrary objects of the sort described in [8]. One would, of course, like to be able to say something a bit more concrete than this in defense of the ontology of stratified models. But the task is quite difficult. This has resulted in a variety of criticisms of stratified models (see [4] for a recent case of this).

I have previously defended stratified semantics from some of these criticisms (see [15]). Here I switch sides and instead point out problems. The basic plan is this: stratified models have the feel of oddly-packaged hyperdoctrines. But if we repackage stratified models in a way that plays up their hyperdoctrinality, rather than bringing the blurily-glimpsed arbitrary objects into sharper focus, we instead find that their base category seems to reflect no coherent ontology at all. Thus, if presenting a theory as a hyperdoctrine in general limns the ontology of the theory, we have very good reason to be worried about the ontology of stratified semantics. This leaves open the possibility of a 'redemption' of sorts: perhaps the repackaging I present here is, despite being the natural option, not the correct one. But we will have to leave investigation of that question to another time.

Given that stratified semantics was presented as a general purpose semantic theory for a broad range of relevant logics, and given that the purpose of the project is to show a problem with stratified semantics, there is no reason to examine a broad range of logics. After all, if there is a problem with its implementation in one of the logics it was meant to cover, that suffices to demonstrate an issue with the semantics as a whole. So we will focus attention here on the logic RWQ. This restriction is motivated by the simple fact that RW occupies a convenient 'middle' position in the usual lattice of relevance logics (see, e.g. the diagram in $\$ 4.3$ of [21]) and the fact that, in the author's experience, RW tends to be a simpler logic to work with than any of the obvious alternatives.

The paper proceeds as follows. In $\S 2$, we cover necessary preliminaries, including the definition of the logic RWQ. In $\S 3$, we present a version stratified semantics. Soundness and completeness for RWQ with respect to the version of stratified semantics I present are given in the appendix. $\$ 4$ introduces the basic notion of an RW hyperdoctrine. In $\S 5$, we prove that RWQ is sound with respect to a hyperdoctrinal semantics. In $\S 6$, we provide a way to build a hyperdoctrine $\mathbf{h y p}^{\mathbf{T}}$ corresponding to any stratified model $\mathbf{T}$, and show that $\mathbf{h y p}^{\mathbf{T}}$ and $\mathbf{T}$ make true exactly the same sentences. Completeness of the hyperdoctrinal semantics is then an immediate corollary of completeness for the stratified semantics. $\$ 7$ gives a philosophical conclusion. 


\section{Preliminaries}

We work in a standard first-order language $\mathscr{L}$ with primitive connectives $\neg, \wedge$, and $\rightarrow$, and with $\forall$ as its only quantifier. Disjunction is defined in the usual way: $\phi \vee \psi:=\neg(\neg \phi \wedge \neg \psi)$. We will take $\mathscr{L}$ to have countably many variables $v_{1}, v_{2}, \ldots$, and take Var to be the set of variables. We will typically use variants of $v, w, x, y$, and $z$ as metavariables ranging over variables. For $X \subseteq$ Var, we let $\mathscr{L}_{X}$ be the set of wffs whose free variables come from $X$. Note in particular that it follows from these definitions that $\mathscr{L}_{\emptyset}$ is the set of sentences and that if $X \subseteq Y$, then $\mathscr{L}_{X} \subseteq \mathscr{L}_{Y}$.

Following Ross Brady (see [3]) we identify RWQ as the smallest set of wffs containing every $\mathscr{L}$-instance of the following twelve axioms which is also closed under all three of the listed rules.

$$
\begin{aligned}
& \text { A1 } \alpha \rightarrow \alpha \\
& \text { A2 }(\alpha \wedge \beta) \rightarrow \alpha \\
& \text { A3 }(\alpha \wedge \beta) \rightarrow \beta \\
& \text { A4 }((\alpha \rightarrow \beta) \wedge(\alpha \rightarrow \gamma)) \rightarrow(\alpha \rightarrow(\beta \wedge \gamma)) \\
& \text { A5 }(\alpha \wedge(\beta \vee \gamma)) \rightarrow((\alpha \wedge \beta) \vee(\alpha \wedge \gamma)) \\
& \text { A6 } \neg \neg \rightarrow \alpha \\
& \text { A7 }(\alpha \rightarrow \neg \beta) \rightarrow(\beta \rightarrow \neg \alpha) \\
& \text { A8 }(\alpha \rightarrow \beta) \rightarrow((\beta \rightarrow \gamma) \rightarrow(\alpha \rightarrow \gamma)) \\
& \text { A9 } \alpha \rightarrow((\alpha \rightarrow \beta) \rightarrow \beta) \\
& \text { A10 } \forall x \phi \rightarrow \phi(y / x) \text { where } y \text { is free for } x \text { in } \phi . \\
& \text { A11 } \forall x(\phi \rightarrow \psi) \rightarrow(\phi \rightarrow \forall x \psi) \text { where } x \text { is not free in } \phi . \\
& \text { A12 } \forall x(\phi \vee \psi) \rightarrow(\phi \vee \forall x \psi) \text { where } x \text { is not free in } \phi . \\
& \text { R1 } \frac{\alpha, \alpha \rightarrow \beta}{\beta} \\
& \text { R2 } \frac{\alpha, \beta}{\alpha \wedge \beta} \\
& \text { R3 } \frac{\phi}{\forall x \phi}
\end{aligned}
$$

For more detail on RWQ or its place in the logical landscape, see [1], [17], or [21].

\section{Stratified Semantics}

The semantics I give here differs mildly from what is called 'stratified semantics' in [9]. Rather than spending a great deal of time comparing and contrasting the theories, I instead directly prove in the appendix that RWQ is sound and complete for the semantics I give. I take it to be clear on inspection, however, that the theory I give is similar enough to Fine's to deserve the 'stratified' moniker. 
Definition 1. A frame is a 6-tuple $\left\langle T, \cdot, \ell, \leq, P,{ }^{*}\right\rangle$ with $\langle T, \cdot\rangle$ a commutative monoid, $\ell$ an identity element for $\langle T, \cdot\rangle, \leq$ a partial ordering of $T, P \subseteq T$, and $*: P \longrightarrow P$, all of which must satisfy the following restrictions:

F1 If $s \leq t$, then $s \cdot u \leq t \cdot u$.

F2 If $p \in P$ and $s \cdot t \leq p$ then there are $q_{1}$ and $q_{2}$ in $P$ so that $s \leq q_{1}$ and $q_{1} \cdot t \leq p$, and $t \leq q_{2}$ and $s \cdot q_{2} \leq p$.

F4 If $t \cdot p \leq q$, then $t \cdot q^{*} \leq p^{*}$.

F5 $p^{* *}=p$.

We will tend to omit ' ' and write monoid multiplication using concatenation.

One well-known example of a frame takes $T$ to be the set of theories in an appropriate logic; $t_{1} t_{2}=\left\{B: A \rightarrow B \in t_{1}\right.$ and $\left.A \in t_{2}\right\}$ (in [25] this is called the application of $t_{1}$ to $t_{2}$ ); $\ell$ to be the logic itself (that is, the set of theorems); $\leq$ to be the usual set-theoretic containment relation; $P$ to be the set of prime theories; and $t^{*}=\{\phi: \neg \phi \notin t\}$ (that is, $*$ is the so-called 'Routley star'). ${ }^{3}$

Definition 2. A z-model is a triple $\langle F$, Dom, $I\rangle$, where

- $F=\left\langle T, \cdot, \ell, \leq, P,{ }^{*}\right\rangle$ is a frame,

- Dom is a nonempty set (the domain),

- $\quad I$ is function mapping variables to Dom and $n$-ary predicates to functions from $T$ to subsets of Dom ${ }^{n}$. We require that $I$ satisfy two conditions:

- If $s \leq t$, then $I(s)(R) \subseteq I(t)(R)$.

$-\bigcap_{t \leq p \in P} I(p)(R)=I(t)(R)$

Definition 3. If $G=\langle F$, Dom, $I\rangle$ is a z-model, $t \in F$, and $a$ and $b$ are distinct members of Dom, then we say $t$ is symmetric in $a$ and $b$ when for all $R,\left\langle d_{1}, \ldots, d_{k-1}, a, d_{k+1}, \ldots, d_{n}\right\rangle \in$ $I(t)(R)$ iff $\left\langle d_{1}, \ldots, d_{k-1}, b, d_{k+1}, \ldots, d_{n}\right\rangle \in I(t)(R)$.

Definition 4. A stratified model consists of the following data:

- For each finite $X \subseteq$ Var, a z-model $\mathbf{T}(X)=\left\langle T_{X},{ }_{X}, \ell_{X}, \leq_{X}, P_{X}, *_{X}, \operatorname{Dom}_{X}, I_{X}\right\rangle$ and a function mapping each pair $\langle a, b\rangle \in \operatorname{Dom}_{X} \times \operatorname{Dom}_{X}$ to a closure operator ${ }^{a}[-]_{X}^{b}$ : $T_{X} \longrightarrow T_{X}{ }^{4}$

- For each finite $X \subseteq Y$, monotonic functions $\downarrow_{X}^{Y}: T_{Y} \rightarrow T_{X}$ and $\uparrow_{X}^{Y}: T_{X} \rightarrow T_{Y}$.

We require that these data satisfy the following conditions:

SM1 If $X \subseteq Y$, then $\operatorname{Dom}_{X} \subseteq \operatorname{Dom}_{Y}$.

$\mathrm{SM} 2{ }^{a}[t]_{X}^{b}$ is symmetric in $a$ and $b$; if $t$ is already symmetric in $a$ and $b$, then ${ }^{a}[t]_{X}^{b}=t$.

\footnotetext{
${ }^{3}$ The Routley star was introduced by Routley and Routley in [23]. For more on how to interpret it, see [6] or [22]. An anonymous referee helpfully points out that there are precursors to the Routley star in the history of logic, some of which are helpfully surveyed in [5, §4.3-4.4]. Here, Dunn amusingly reports that [23] 'more or less just springs [the star operator] on the reader'.

${ }^{4}$ Since ${ }^{a}[-]_{X}^{b}$ is a closure operator, we have that, $t \leq{ }^{a}[t]_{X}^{b}$ for all $t$; if $t \leq u$ then ${ }^{a}[t]_{X}^{b} \leq{ }^{a}[u]_{X}^{b}$; and ${ }^{a}\left[{ }^{a}[t]_{X}^{b}\right]_{X}^{b}={ }^{a}[t]_{X}^{b}$.
} 
SM3 If $p \in P_{X}$, then ${ }^{a}[p]_{X}^{b} \in P_{X}$ and $\left({ }^{a}[p]_{X}^{b}\right)^{*}$ is symmetric in $a$ and $b$.

$\mathrm{SM} 4{ }^{a}[t u]_{X}^{b} \leq{ }^{a}[t]_{X}^{b}{ }^{a}[u]_{X}^{b} \leq{ }^{a}[t]_{X}^{b} u$.

SM5 If ${ }^{a}[s]_{X}^{b} \leq p \in P_{X}$, then there is a $t$ with $s \leq t,{ }^{a}[t]_{X}^{b} \leq p$ and ${ }^{a}[t]_{X}^{b} \in P_{X}$.

SM6 $\ell_{X} \uparrow_{X}^{Y}=\ell_{Y}$.

SM7 $t \downarrow_{Y}^{Z} \downarrow_{X}^{Y}=t \downarrow_{X}^{Z} ; t \uparrow_{X}^{Y} \uparrow_{Y}^{Z}=t \uparrow_{X}^{Z}$

SM8 $t \uparrow_{X}^{Y} \downarrow_{X}^{Y}=t$,

SM9 If $X \cap W=Y$, then $t \downarrow_{Y}^{X} \uparrow_{Y}^{W}=t \uparrow_{X}^{V} \downarrow_{W}^{V}$ for all $V \supseteq X \cup W$.

SM10 $t \downarrow_{X}^{Y} \uparrow_{X}^{Y} \leq t$.

SM11 $(t u) \uparrow_{X}^{Y}=t \uparrow_{X}^{Y} u \uparrow_{X}^{Y}$.

SM12 $\left(t u \uparrow_{X}^{Y}\right) \downarrow_{X}^{Y} \leq t \downarrow_{X}^{Y} u$.

SM13 If $x \in X \cap Y$, then $I_{X}(x)=I_{Y}(x){ }^{5}$

SM14 If $R$ is $n$-ary, then $I_{Y}(t)(R) \cap \operatorname{Dom}_{X}^{n}=I_{X}\left(t \downarrow_{X}^{Y}\right)(R)$.

SM15 If $t \downarrow_{X}^{Y} \leq p \in P_{X}$ then for some $q \in P_{Y}, t \leq q$ and $q \downarrow_{X}^{Y}=p$.

SM16 $P_{Y} \downarrow_{X}^{Y} \subseteq P_{X}$ and for $p \in P_{Y}, p^{*} \downarrow_{X}^{Y}=\left(p \downarrow_{X}^{Y}\right)^{*}$.

SM17 If $a$ and $b$ are in $\operatorname{Dom}_{X}$ then ${ }^{a}\left[t \uparrow_{X}^{Y}\right]_{Y}^{b}={ }^{a}[t]_{X}^{b} \uparrow_{X}^{Y}$.

SM18 If $a \in \operatorname{Dom}_{Y}-\operatorname{Dom}_{X}$ and $b \in \operatorname{Dom}_{X}$, then ${ }^{a}\left[t \uparrow_{X}^{Y}\right]_{Y}^{b} \downarrow_{X}^{Y} \leq t$.

If $\mathbf{T}$ is a stratified model, $X \subseteq \operatorname{Var}$ is finite, $\phi \in \mathscr{L}_{X}$, and $t \in \mathbf{T}(X)$, then we define $\mathbf{T}, X, t \vDash \phi$ as follows:

- $\mathbf{T}, X, t \vDash P x_{1} \ldots x_{n}$ iff $\left\langle I_{X}\left(x_{1}\right), \ldots, I_{X}\left(x_{n}\right)\right\rangle \in I_{X}(t)(P)$

- $\mathbf{T}, X, t \vDash \phi \wedge \psi$ iff $\mathbf{T}, X, t \vDash \phi$ and $\mathbf{T}, X, t \vDash \psi$

- $\mathbf{T}, X, t \vDash \neg \phi$ iff $\mathbf{T}, X, p^{*} \not \models \phi$ for all $t \leq p \in P_{X}$.

- $\mathbf{T}, X, t \vDash \phi \rightarrow \psi$ iff for all $u$, if $\mathbf{T}, X, u \vDash \phi$ then $\mathbf{T}, X, t u \vDash \psi$.

- $\mathbf{T}, X, t \vDash \forall x \phi(x)$ iff $\mathbf{T}, Y, t \uparrow_{X}^{Y} \vDash \phi(y)$ for some $Y \supsetneq X$ and $y \in Y-X$.

If $\phi\left(y_{1}, \ldots, y_{n}\right) \notin \mathscr{L}_{X}$ and $y_{1}, \ldots, y_{n}$ are the free variables in $\phi$ that are not in $X$ then $\mathbf{T}, X, t \vDash \phi$ when $\mathbf{T}, X, t \vDash \phi\left(y_{1} / x_{1}, y_{2} / x_{2}, \ldots, y_{n} / x_{n}\right)$ for all $\left\langle x_{1}, \ldots, x_{n}\right\rangle \in X^{n}$.

We say that $\phi$ is valid in $\mathbf{T}$ (and we write $\mathbf{T} \vDash \phi$ ) when $\mathbf{T}, X, \ell_{X} \vDash \phi$ for all $X$. We say $\phi$ is valid in the class of models $\mathbf{K}$ when $\mathbf{T} \vDash \phi$ for every $\mathbf{T} \in \mathbf{K}$. We take Str to be the class of all stratified models. In the appendices we present proofs (lightly modified from those in [9]) showing that $\mathbf{S t r} \vDash \phi$ iff $\phi \in$ RWQ.

For more on stratified models, the reader is encouraged to read [9] or [10]. The only two further resources on stratified semantics that I am aware of are [16] and [15]. To best understand stratified models, it is useful to contrast them with the alternative semantics presented in [18], which has been further examined by Shawn Standefer in as-yet unpublished material.

\footnotetext{
${ }^{5}$ Context will in general suffice to determine whether we mean, e.g. $I_{X}(x)$ or $I_{Y}(x)$, and where context doesn't so determine this feature guarantees that it doesn't matter. So we will tend to just write $I(x)$.
} 


\section{RW hyperdoctrines}

As noted, there is little standardization when it comes to hyperdoctrines. So it's worthwhile to state explicitly that the definition we will use is modeled on the definition found in [19], which loosely follows [20] and [12].

In general, things that are called hyperdoctrines are contravariant functors from a category that has finite products to a category of small categories. The image of said functor is usual required to be contained in the category of algebras that provide algebraic models of the propositional version of whatever logic we're interested in. Quantifiers then arise naturally as adjoints to the functorial images of the arrows in the base category.

We also mentioned in the introduction that the base category is typically taken to have as its objects the types in whatever language we are interpreting. Since $\mathscr{L}$ has a single sort of object, $\mathscr{L}$ 's type theory is rather boring: for each $n \geq 1$, it has a type consisting of $n$-tuples of members of the domain, and that's it. So it's natural to identify $\mathscr{L}$ 's types with the category $\mathbf{T}_{\mathscr{L}}$ that has (nonempty) finite products and is generated by a single object. The arrows in $\mathbf{T}_{\mathscr{L}}$ then correspond in a natural way to the actual terms - that is, the strings of variables. Modulo a little fiddling, all this is exactly as described in [24].

Familiar as $\mathbf{T}_{\mathscr{L}}$ is, $\mathbf{T}_{\mathscr{L}}^{\text {op }}$ is yet more familiar: it's the category with finite coproducts generated by one object, which is just the category of (nonzero) finite ordinals. And since a contravariant functor with domain $\mathbf{T}_{\mathscr{L}}$ is the same thing as a covariant functor with domain $\mathbf{T}_{\mathscr{L}}^{\text {op }}$, one natural way to present an $\mathscr{L}$-hyperdoctrine is as a covariant functor FinOrd ${ }^{+} \longrightarrow$ Cat (where FinOrd ${ }^{+}$is the category of (nonzero) finite ordinals and inclusions) satisfying some conditions.

These considerations give us reason to be ok with covariant hyperdoctrines. But it does leave us expecting the base category to be something nice like FinOrd. But the natural choice of base category for a hyperdoctrinal version of stratified semantics is not so nice.

Intuitively, the operation ' $\uparrow_{X}^{Y}$ ' in a stratified model represents the inclusion of $\mathscr{L}_{X}$ into $\mathscr{L}_{Y}$, while the operation ${ }^{a}[-]_{Y}^{b}$ represents represents the imposition of some sort of equivalence relation on the domain and corresponding varieties of equivariance on the other machinery. So, intuitively, a category capturing this should have as its objects the finite subsets of Var and as arrows everything generated by the inclusion and 'impose an equivalence relation' operations. That's a rather vague and inchoate motivation; nonetheless it suffices as a motivation for taking our hyperdoctrines to have the category $\mathbf{E q R} \mathbf{R}$, which we now define, as their domain:

Definition 5. We define the category EqRIn as follows:

- The objects of EqRIn are the finite sets of variables.

- The arrows of EqRIn are triples $\langle X, Y, \sim\rangle$ where $X \subseteq Y$ and $\sim$ is an equivalence relation on $Y$. Such a triple is a morphism from $X$ to $Y$.

- If $\left\langle Y, Z, \sim_{1}\right\rangle$ and $\left\langle X, Y, \sim_{2}\right\rangle$ are composable arrows, then their composition is $\left\langle X, Z, \sim_{3}\right\rangle$, where $\sim_{3}$ is the transitive closure of the relation $\sim_{3}^{\prime}$ that we define by saying $x \sim_{3}^{\prime} y$ iff either $x \sim_{1} y$ or $x \sim_{2} y$. 
- It follows that the triple $\left\langle X, X, \mathrm{id}_{X}\right\rangle$ (where $\mathrm{id}_{X}$ is the identity relation on $X$ ) thus plays the role of the identity arrow at $X$.

It's not completely obvious that composition in EqRIn is associative, or that the relation $\sim_{3}$ will in general be symmetric. We'll pause to prove the former; similar techniques can be used to prove the latter.

Lemma 1. If $f$, $g$, and $h$ are arrows in EqRIn, then $(f \circ g) \circ h=f \circ(g \circ h)$

Proof. Suppose $z_{1} \sim(f \circ g) \circ h z_{2}$. Then there are $y_{1}, \ldots, y_{n}$ so that $y_{1}=z_{1}, y_{n}=z_{2}$, and $y_{1} \sim_{(f \circ g) \circ h}^{\prime} y_{2} \sim_{(f \circ g) \circ h}^{\prime} \cdots \sim_{(f \circ g) \circ h}^{\prime} y_{n}$. Thus for all $1 \leq i \leq n-1$, either $y_{i} \sim_{f \circ g}^{\prime} y_{i+1}$ or $y_{1} \sim_{h} y_{i+1}$. Suppose that in fact for $k \in K, y_{i_{k}} \sim_{f \circ g} y_{i_{k}+1}$ while for $j \notin K, y_{i_{j}} \sim_{h}$ $y_{i_{j}+1}$. Then for each $k \in K$, there are $y_{1}^{k} \ldots y_{m_{k}}^{k}$ with $y_{i_{k}}=y_{1}^{k}$ and $y_{i_{k}+1}=y_{m_{k}}^{k}$ and $y_{1}^{k} \sim_{f \circ g}^{\prime} y_{2}^{k} \sim_{f \circ g}^{\prime} \cdots \sim_{f \circ g}^{\prime} y_{m_{k}}^{k}$. Thus, for each $1 \leq l \leq m_{k}-1$, either $y_{l}^{k} \sim_{f} y_{l+1}^{k}$ or $y_{l}^{k} \sim g y_{l+1}^{k}$.

But if $y_{l}^{k} \sim_{g} y_{l+1}^{k}$, then $y_{l}^{k} \sim_{g \circ h} y_{l+1}^{k}$. So for each $1 \leq l \leq m_{k}-1$, either $y_{l}^{k} \sim_{f} y_{l+1}^{k}$ or $y_{l}^{k} \sim_{g \circ h} y_{l+1}^{k}$. Thus for each $k \in K, y_{i_{k}} \sim_{f \circ(g \circ h)}^{\prime} y_{i_{k}+1}$. Also, for $j \notin K$, since $y_{i_{j}} \sim_{h}$ $y_{i_{j}+1}, y_{i_{j}} \sim_{g \circ h} y_{i_{j}+1}$, and thus $y_{i_{j}} \sim_{f \circ(g \circ h)} y_{i_{j}+1}$. Thus $z_{1} \sim_{f \circ(g \circ h)} z_{2}$.

A similar argument gives the converse.

Some further notes:

- We identify the inclusion $X \hookrightarrow Y$ with the triple $\left\langle X, Y, \operatorname{id}_{Y}\right\rangle$.

- If $f: X \longrightarrow Y$, we will write $\sim_{f}$ for the equivalence relation associated to $f$, write $\left.f\right|_{X}$ for the obvious restriction of $f$ to an arrow $X \longrightarrow X$, and write $f^{+}$for the obvious extension of $f$ to an arrow $Y \longrightarrow Y$.

- The reader familiar with hyperdoctrinal matters is likely to have grave concerns about using EqRIn as our base category. These concerns are entirely warranted. We will return to discuss this matter in $§ 7$.

One more difference between hyperdoctrines as I defined here and hyperdoctrines as defined elsewhere is worth noting. Typically, in a hyperdoctrine left adjoints of certain arrows semantically correspond to existential quantification and right adjoints of these same arrows semantically correspond to universal quantification. However, a convenient feature of the axiomatization of RWQ given in $\S 2$ is that it requires only universal quantification. This allows us to shorten our definition by stating all required features in terms of right adjoints alone. Finally, since (as is proved in, e.g. [7], a slightly corrected version of which can be found in [2]) frames give a natural quasi-algebraic semantics for RW, we will map to the category Frame whose objects are frames and whose arrows (the frame morphisms) we define as follows:

Definition 6. If $F$ and $G$ are frames, then a monotonic function $\mu: F \rightarrow G$ is a frame morphism when (a) for all $t$ and $u$ in $F, \mu(t u) \leq \mu(t) \mu(u)$, and (b) if $\mu(t) \leq p \in P_{G}$, then $\mu(t) \leq \mu(q) \leq p$ for some $t \leq q \in P_{F}$. We say a frame morphism is exact when $\mu(t u)=\mu(t) \mu(u)$.

Definition 7. An $R$ W-hyperdoctrine is a functor $H: \mathbf{E q R I n} \longrightarrow$ Cat such that 
RH1 The image of $H$ is in Frame.

RH2 For each $f: X \longrightarrow X$, if $t \in H(X)$ then $t \leq H_{f}(t)$; if $p \in P_{X}$, then $H_{f}(p) \in P_{X}$; $H_{f}\left(t H_{f}(u)\right)=H_{f}(t) u$; and $H_{f}\left(H_{f}(p)^{*}\right)=H_{f}(p)^{*}$.

RH3 For each inclusion $\imath=\left\langle X, Y, \operatorname{id}_{Y}\right\rangle, H_{l}$ is exact and has a right adjoint $\Pi_{l}$ : $H(Y) \longrightarrow H(X)$. We will generally write these morphisms $H_{X \subseteq Y}$ and $\Pi_{X \subseteq Y}$.

RH4 For all $f: X \longrightarrow Y, \Pi_{X \subseteq Y}\left(H_{f}(t)\right) \leq H_{\left.f\right|_{X}}(t) ; \Pi_{X \subseteq Y}\left(P_{Y}\right) \subseteq P_{X} ;$ and $\Pi_{X \subseteq Y}\left(p^{*}\right)=$ $\left(\Pi_{X \subseteq Y}(p)\right)^{*}$.

RH5 If $Y \subseteq Z$ and $X \cap Z=\emptyset$, then the following commutes:

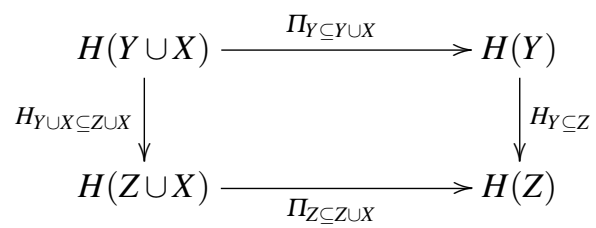

RH6 If $s \in H(X)$ and $t \in H(Y)$, then $\Pi_{X \subseteq Y}\left(H_{X \subseteq Y}(s) t\right) \leq s \Pi_{X \subseteq Y}(t){ }^{6}$

Lemma 2. $\Pi_{Y \subseteq Z} \circ \Pi_{X \subseteq Y}=\Pi_{X \subseteq Z}$

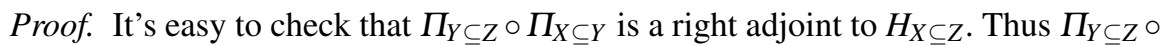
$\Pi_{X \subseteq Y}$ and $\Pi_{X \subseteq Z}$ are isomorphic. But since everything in sight is a poset, this just means that they're identical.

Some notational conventions will be useful: we will write $\bar{x}$ and $\bar{y}$ for strings of variables. Given a string of variables $\bar{x}=x_{1} x_{2} \ldots x_{n}$, we will write $|\bar{x}|$ for its underlying set; that is for $\left\{x_{1}, x_{2}, \ldots, x_{n}\right\}$ in this case. If $f: X \longrightarrow Y$, then we say $\bar{x} \sim_{f} \bar{y}$ iff $x_{i} \sim f y_{i}$ for all $i$.

Definition 8. Given an RW hyperdoctrine $H$, an assignment for $H$ is a function $\alpha$ mapping each atomic formula $R \bar{x}$ and finite set of variables $X$ to a set of theories $\alpha(X, R \bar{x})$. We require that assignments satisfy the following conditions:

A1 If $s \in \alpha(X, R \bar{x})$ and $s \leq t$ then $t \in \alpha(X, \bar{x})$.

A2 If $\left\{p: t \leq p \in P_{X}\right\} \subseteq \bar{\alpha}(X, R \bar{x})$, then $t \in \alpha(X, R \bar{x})$.

A3 If $f: X \longrightarrow Y$, then $H_{f}(t) \in \alpha(Y, R \bar{y})$ iff $H_{f}(t) \in \alpha(Y, R \bar{z})$ for all $\bar{z} \sim_{f} \bar{y}$.

A4 If $|\bar{x}| \subseteq X$, then $\Pi_{X \subseteq Y}(t) \in \alpha(X, R \bar{x})$ iff $t \in \alpha(Y, R \bar{x})$.

Lemma 3. $\Pi_{X \subseteq Y}\left(H_{X \subseteq Y}(t)\right)=t$

Proof Sketch: By adjointness, $t \leq \Pi(H(t))$, and by RH4, $\Pi(H(t)) \leq H_{\mathrm{id}_{X}}(t)=t$.

Lemma 4. If $|\bar{x}| \in X$, then $t \in \alpha(X, R \bar{x})$ iff $H_{X \subseteq Y}(t) \in \alpha(Y, R \bar{x})$.

\footnotetext{
${ }^{6}$ Category-theorists often call conditions that make mention of features internal to the objects in a category evil. Both RH2 and RH4 have a bit too much 'evil' in them to win broad approbation from categorially-minded folks. I have a sneaking suspicion that they both conditions can, with proper reformulation of other parts of the theory, be avoided or at least made less evil, but have not been able to do so on my own to this point.
} 
Proof Sketch: Apply A4 and the previous lemma.

Lemma 5. If $f: X \rightarrow Y$, then if $H_{X \subseteq Y}(t) \in \alpha(Y, R \bar{y})$, then $H_{f}(t) \in \alpha(Y, R \bar{y})$ as well.

Proof. To begin, notice that we can rewrite $f$ as the inclusion $X \subseteq Y$ followed by $f^{+}=\left\langle Y, Y, \sim_{f}\right\rangle$. Thus $H_{f}(t)=H_{f^{+}}\left(H_{X \subseteq Y}(t)\right)$. So by RH2, $H_{X \subseteq Y}(t) \leq H_{f}(t)$, and thus by A1, if $H_{X \subseteq Y}(t) \in \alpha(Y, R \bar{y})$, then $H_{f}(t) \in \alpha(Y, R \bar{y})$ as required.

For $\phi \in \mathscr{L}_{X}$, we define truth in $H$ relative to the set $X$, the theory $t$, and the assignment $\alpha$ as follows:

- $H, X, t, \alpha \Vdash R \bar{x}$ iff $t \in \alpha(X, R \bar{x})$.

- $H, X, t, \alpha \Vdash \phi \wedge \psi$ iff $H, X, t, \alpha \Vdash \phi$ and $H, X, t, \alpha \Vdash \psi$.

- $H, X, t, \alpha \Vdash \phi \rightarrow \psi$ iff for all $u$, if $H, X, u, \alpha \Vdash \phi$, then $H, X, t u, \alpha \Vdash \psi$.

- $H, X, t, \alpha \Vdash \neg \phi$ iff $H, X, p^{*}, \alpha \Vdash \phi$ for all $t \leq p \in P_{Y}$.

- $H, X, t, \alpha \Vdash \forall x \phi(x)$ iff for some $y \notin X, H, X \cup\{y\}, H_{X \subseteq X \cup\{y\}}(t), \alpha \Vdash \phi(y)$.

Let $X \subsetneq Y,|\bar{x}| \subseteq X$ and $|\bar{y}| \subseteq Y-X$. Then $H, X, t, \alpha \Vdash \phi(\overline{x y})$ iff for all $H, X, t, \alpha \Vdash$ $\phi(\overline{x z})$ for all appropriate-length sequences $\bar{z}$ with $|\bar{z}| \subseteq X$.

In general, we will write $X, t \Vdash \phi$ in place of $H, X, t, \alpha \Vdash \phi .^{7}$ We say that $\phi$ is valid in $H$ relative to $\alpha$ (and write $H, \alpha \Vdash \phi$ ) when $H, X, \ell_{X}, \alpha \Vdash \phi$ for all $X$. We say $\phi$ is valid in $H$ (and write $H \Vdash \phi$ ) when $H, \alpha \Vdash \phi$ for all $\alpha$. We say that $\phi$ is valid (and write hyp $\Vdash \phi$ ) when $H \Vdash \phi$ for all $H$. Note that we will use ' $\vDash$ ' when discussing matters related to stratified models and use ' $\Vdash$ ' for the corresponding hyperdoctrinal matters.

The plan for the remainder of the paper is this: we will first prove soundness of RWQ for RW hyperdoctrines. Then we will show how to repackage any given stratified model as an equivalent (in the sense specified below in Theorem 3) RW hyperdoctrine. Completeness for the hyperdoctrinal semantics is an immediate consequence of this theorem.

\section{Soundness for RW hyperdoctrines}

In this section we show that if $\phi \in \mathrm{RWQ}$, then hyp $\Vdash \phi$. Throughout, let $H$ be an RW hyperdoctrine, $\alpha$ be an assignment for $H$, and $X$ be a finite set of variables. We use the following abbreviations: $\mathrm{IH}={ }_{\text {def }}$ the inductive hypothesis; $\mathrm{OH}={ }_{\text {def }}$ order heredity (Lemma 6); $\mathrm{AH}=_{\text {def }}$ adjoint heredity (Lemma 8).

Lemma 6 (Order Heredity). If $s \leq t$ then $X, s \Vdash \phi$ only if $X, t \Vdash \phi$.

Proof. By a straightforward induction on $\phi$.

Lemma 7. If $f: X \rightarrow Y$, then $Y, H_{f}(t) \Vdash \phi(\bar{y})$ iff $Y, H_{f}(t) \Vdash \phi(\bar{z})$ for all $\bar{z} \sim_{f} \bar{y}$.

${ }^{7}$ The $Y$ 's contain the kind of information (viz. where in the hyperdoctrine one is doing the evaluating) that is easy to lose track of, so we will in general not omit them. 
Proof. By induction on $\phi$. For atoms, the result is immediate from Lemma 5. The 'if' direction is trivial for all cases; as are both directions of the conjunction case. We consider the 'only if' directions for the remaining cases below.

Suppose $Y, H_{f}(t) \Downarrow \neg \psi(\bar{z})$ for some $\bar{z} \sim_{f} \bar{y}$. Then $Y, p^{*} \Vdash \psi(\bar{z})$ for some $H_{f}(t) \leq$ $p \in P_{Y}$. Since $H_{f}$ is a frame morphism, there is a $t \leq q \in P_{Y}$ with $H_{f}(t) \leq H_{f}(q) \leq$ $p \in P_{Y}$. Since $H_{f}(q) \leq p, p^{*} \leq H_{f}(q)^{*}$. Thus $Y, H_{f}(q)^{*} \Vdash \psi(\bar{z})$. Recall from the proof of Lemma 5 that $H_{f}=H_{f^{+}} \circ H_{X \subseteq Y}$. Using RH2 we have that $H_{f^{+}}\left(H_{f}(q)^{*}\right)=$ $H_{f^{+}}\left(H_{f^{+}}\left(H_{X \subseteq Y}(q)\right)^{*}\right)=H_{f^{+}}\left(H_{X \subseteq Y}(q)\right)^{*}=H_{f}(q)^{*}$. So by IH, $Y, H_{f}(q)^{*} \Vdash \psi(\bar{w})$ for all $\bar{w} \sim_{f} \bar{f}$. Thus in particular $Y, H_{f}(q)^{*} \Vdash \psi(\bar{y})$. So $Y, H_{f}(t) \forall \neg \psi(\bar{y})$.

Suppose $Y, H_{f}(t) \Downarrow \psi_{1}(\bar{z}) \rightarrow \psi_{2}(\bar{z})$ for some $\bar{z} \sim_{f} \bar{y}$. Then there is a $u$ so that $Y, u \Vdash \psi_{1}(\bar{z})$ but $Y, H_{f}(t) u \Downarrow \nvdash \psi_{2}(\bar{z})$. As before we write $H_{f}=H_{f^{+}} \circ H_{X \subseteq Y}$. Now observe that

$$
\begin{aligned}
H_{f}(t) u & =H_{f^{+}}\left(H_{X \subseteq Y}(t)\right) u \\
& =H_{f^{+}}\left(H_{f^{+}}\left(H_{X \subseteq Y}(t)\right)\right) u \\
& =H_{f^{+}}\left(H_{f^{+}}\left(H_{X \subseteq Y}(t)\right) H_{f^{+}}(u)\right)
\end{aligned}
$$

Thus by IH, $Y, H_{f}(t) u \Vdash \forall \psi_{2}(\bar{y})$. But also note that

$$
\begin{aligned}
H_{f}(t) u & =H_{f^{+}}\left(H_{f^{+}}\left(H_{X \subseteq Y}(t)\right) H_{f^{+}}(u)\right) \\
& \geq H_{f^{+}}\left(H_{X \subseteq Y}(t)\right) H_{f^{+}}(u) \\
& =H_{f}(t) H_{f^{+}}(u)
\end{aligned}
$$

So by OH, $Y, H_{f}(t) H_{f^{+}}(u) \Vdash \psi_{2}(\bar{y})$. But since $u \leq H_{f^{+}}(u)$, we also have $Y, H_{f^{+}}(u) \Vdash$ $\psi_{1}(\bar{z})$. So by IH, $Y, H_{f}(u) \Vdash \psi_{1}(\bar{y})$. Thus $Y, H_{f}(t) \Vdash \psi_{1}(\bar{y}) \rightarrow \psi_{2}(\bar{y})$.

For universals, $Y, H_{f}(t) \Vdash \forall x \psi(\bar{y} x)$ iff for some $w \notin Y, Y \cup\{w\}, H_{Y \subseteq Y \cup\{w\}}\left(H_{f}(t)\right) \Vdash$ $\psi(\bar{y} w)$. Let $l_{1}$ be the inclusion $\left\langle Y, Y \cup\{w\}, \operatorname{id}_{Y \cup\{w\}}\right\rangle$, and $\imath_{2}$ be the inclusion $\langle X, X \cup$ $\left.\{w\}, \operatorname{id}_{X \cup\{w\}}\right\rangle$. Finally, let $\sim_{f \oplus i d_{\{w\}}}$ be the equivalence relation on $Y \cup\{w\}$ generated by $\sim_{f}$ and $\operatorname{id}_{\{w\}}$ and let $f \oplus \operatorname{id}_{\{w\}}=\left\langle X \cup\{w\}, Y \cup\{w\}, \sim_{f \oplus d_{\{w\}}}\right\rangle$. The key observation is that $\left.\iota_{1} \circ f=\left(f \oplus \operatorname{id}_{\{} w\right\}\right) \circ l_{2}$. Thus $H_{Y \subseteq Y \cup\{w\}}\left(H_{f}(t)\right)=H_{f \oplus \operatorname{id}_{\{w\}}}\left(H_{X \subseteq X \cup\{w\}}(t)\right)$. Thus $Y, H_{f}(t) \Vdash \forall x \psi(\bar{y} x)$ iff for some $w \notin Y, Y \cup\{w\}, H_{f \oplus \operatorname{id}_{\{w\}}}\left(H_{X \subseteq X \cup\{w\}}(t)\right) \Vdash$ $\psi(\bar{y} w)$ iff (by IH) for some $w \notin Y, Y \cup\{w\}, H_{f \oplus i \mathrm{id}_{\{w\}}}\left(H_{X \subseteq X \cup\{w\}}(t)\right) \Vdash \psi(\bar{z} w)$ for all $\bar{z} \sim_{f} \bar{y}$ iff for some $w \notin Y, Y \cup\{w\}, H_{Y \subseteq Y \cup\{w\}}\left(H_{f}(t)\right) \Vdash \psi(\bar{z} w)$ for all $\bar{z} \sim_{f} \bar{y}$ iff $Y, H_{f}(t) \Vdash \forall x \psi(\bar{z} x)$ for all $\bar{z} \sim_{f} \bar{y}$.

Lemma 8 (Adjoint Heredity). If $\phi \in \mathscr{L}_{X}$, then $X, \Pi_{X \subseteq Y}(t) \Vdash \phi$ iff $Y, t \Vdash \phi$.

Proof. By induction on $\phi$. The atomic case follows from A4. The conjunction and negation cases are left to the reader. For conditionals, suppose $Y, t \Vdash \phi \rightarrow \psi$ and let $X, u \Vdash \phi$. By Lemma $3, X, \Pi_{X \subseteq Y}\left(H_{X \subseteq Y}(u)\right) \Vdash \phi$. So by IH, $Y, H_{X \subseteq Y}(u) \Vdash \phi$. So $Y, t H_{X \subseteq Y}(u) \Vdash \psi$. By IH $X, \Pi_{X \subseteq Y}\left(t H_{X \subseteq Y}\right) \Vdash \psi$. But by RH6 and commutativity, $\Pi_{X \subseteq Y}\left(t H_{X \subseteq Y}\right) \leq \Pi_{X \subseteq Y}(t) u$. So by OH, $X, \Pi_{X \subseteq Y}(t) u \Vdash \psi$. Thus $X, \Pi_{X \subseteq Y}(t) \Vdash \phi \rightarrow$ $\psi$.

Now suppose $X, \Pi_{X \subseteq Y}(t) \Vdash \phi \rightarrow \psi$. Let $Y, u \Vdash \phi$. By IH, $X, \Pi_{X \subseteq Y}(u) \Vdash \phi$. Thus $X, \Pi_{X \subseteq Y}(t) \Pi_{X \subseteq Y}(u) \Vdash \psi$. So by Lemma 3, $X, \Pi_{X \subseteq Y}\left(H_{X \subseteq Y}\left(\Pi_{X \subseteq Y}(t) \Pi_{X \subseteq Y}(u)\right)\right) \Vdash$ 
$\psi$. Thus by IH, $Y, H_{X \subseteq Y}\left(\Pi_{X \subseteq Y}(t) \Pi_{X \subseteq Y}(u)\right) \Vdash \psi$. But $H_{X \subseteq Y}\left(\Pi_{X \subseteq Y}(t) \Pi_{X \subseteq Y}(u)\right)=$ $H_{X \subseteq Y}\left(\Pi_{X \subseteq Y}(t)\right) H_{X \subseteq Y}\left(\Pi_{X \subseteq Y}(u)\right) \leq t u$. So by OH, $Y, t u \Vdash \psi$. So $Y, t \Vdash \phi \rightarrow \psi$.

Finally, we turn to the universal case. First note that by RH5, the following commutes for any $z \notin Y$ :

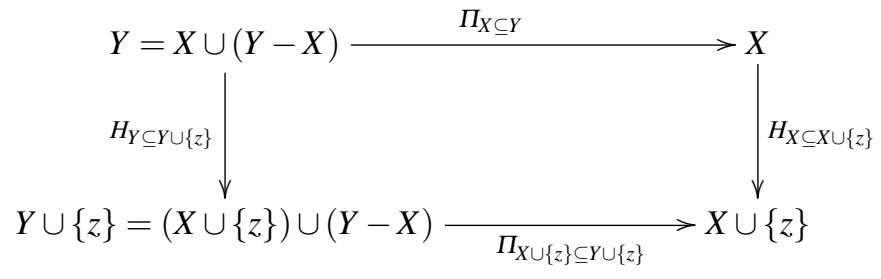

Thus $H_{X \subseteq X \cup\{z\}} \circ \Pi_{X \subseteq Y}=\Pi_{X \cup\{z\} \subseteq Y \cup\{z\}} \circ H_{Y \subseteq Y \cup\{z\}}$. Notice that $Y, t \Vdash \forall x \psi(x)$ iff for some $z \notin Y, Y \cup\{z\}, H_{Y \subseteq Y \cup\{z\}}(t) \Vdash \psi(z)$, iff (by IH) for some $z \notin Y, X \cup$ $\{z\}, \Pi_{X \cup\{z\} \subseteq Y \cup\{z\}}\left(H_{Y \subseteq Y \cup\{z\}}(t)\right) \Vdash \psi(z)$ iff for some $z \notin Y, X \cup\{z\}, H_{X \subseteq X \cup\{z\}}\left(\Pi_{X \subseteq Y}(t)\right) \Vdash$ $\psi(z)$. What remains is to prove that $X, \Pi_{X \subseteq Y}(t) \Vdash \forall x \psi(x)$ iff for some $z \notin Y$, $X \cup\{z\}, H_{X \subseteq X \cup\{z\}}\left(\Pi_{X \subseteq Y}(t)\right) \Vdash \psi(z)$.

The 'if' direction is immediate. For the 'only if' direction, suppose $X, \Pi_{X \subseteq Y}(t) \Vdash$ $\forall x \psi(x)$. Then for some $w \notin X, X \cup\{w\}, H_{X \subseteq X \cup\{w\}}\left(\Pi_{X \subseteq Y}(t)\right) \Vdash \psi(w)$. Choose $w \neq z \notin Y$. Since $\Pi_{X \cup\{w\} \subseteq X \cup\{z, w\}}\left(H_{X \cup\{w\} \subseteq X \cup\{z, w\}}(s)\right)=s$ for all $s$, IH gives that $X \cup$ $\{z, w\}, H_{X \cup\{w\} \subseteq X \cup\{z, w\}}\left(H_{X \subseteq X \cup\{w\}}\left(\Pi_{X \subseteq Y}(t)\right)\right) \Vdash \psi(w)$. Thus $H_{X \subseteq X \cup\{z, w\}}\left(\Pi_{X \subseteq Y}(t)\right) \Vdash$ $\psi(w)$.

Define $\sim_{f}$ so that $z \sim_{f} w, w \sim_{f} z$, and for all $v \in X \cup\{z, w\}, v \sim_{f} v$. Let $f=\left\langle X \cup\{z, w\}, X \cup\{z, w\}, \sim_{f}\right\rangle$ and $f^{-}=\left\langle X, X \cup\{z, w\}, \sim_{f}\right\rangle$. By RH2 and $\mathrm{OH}$, $H_{f}\left(H_{X \subseteq X \cup\{z, w\}}\left(\Pi_{X \subseteq Y}(t)\right)\right) \Vdash \psi(w)$. Thus by the previous lemma, $H_{f}\left(H_{X \subseteq X \cup\{z, w\}}\left(\Pi_{X \subseteq Y}(t)\right)\right) \Vdash$ $\psi(z)$. But clearly $H_{f} \circ H_{X \subseteq X \cup\{z, w\}}=H_{f^{-}}$. Thus by IH, $\Pi_{X \cup\{z\} \subseteq X \cup\{z, w\}}\left(H_{f^{-}}\left(\Pi_{X \subseteq Y}(t)\right)\right) \Vdash$ $\psi(z)$. So by RH4, $\left.H_{\left.f^{-}\right|_{X \cup\{z\}}}\left(\Pi_{X \subseteq Y}(t)\right)\right) \Vdash \psi(z)$. But $\left.H_{f^{-}}\right|_{X \cup\{z\}}=H_{X \subseteq X \cup\{z\}}$, so as required for some $z \notin Y, X \cup\{z\}, H_{X \subseteq X \cup\{z\}}\left(\Pi_{X \subseteq Y}(t)\right) \Vdash \psi(z)$.

Lemma 9. If $X, t \Vdash \forall y \phi(y)$, then $X, t \Vdash \phi(x)$ for all $x \in X$.

Proof. Let $X, t \Vdash \forall y \phi(y)$ and $x \in X$. Let $\sim_{f}$ be the equivalence relation given by the following three conditions: (i) $z \sim_{f} z$ for all $z \in X \cup\{y\}$, (ii) $x \sim_{f} y$, and (iii) $y \sim_{f} x$. Finally, let $f=\left\langle X, X \cup\{y\}, \sim_{f}\right\rangle$. Notice that $H_{f^{+}}\left(H_{X \subseteq X \cup\{y\}}(t)\right)=H_{f}(t)$. So by RH2, $H_{X \subseteq X \cup\{y\}}(t) \leq H_{f}(t)$. Next note that $X \cup\{y\}, H_{X \subseteq X \cup\{y\}}(t) \Vdash \phi(y)$. Thus by OH, $X \cup\{y\}, H_{f}(t) \Vdash \phi(y)$. So by the previous lemma, $X \cup\{y\}, H_{f}(t) \Vdash \phi(x)$. Thus by AH, $X, \Pi_{X \subseteq X \cup\{y\}}\left(H_{f}(t)\right) \Vdash \phi(x)$. So by RH4 and OH, $X, H_{f \mid X}(t) \Vdash \phi(x)$, which is to say $X, t \Vdash \phi(x)$.

Lemma 10. $X, t \Vdash \phi$ iff $X, p \Vdash \phi$ for all $t \leq p \in P_{X}$.

Proof. One direction follows from $\mathrm{OH}$; the other from a straightforward induction on $\phi$.

Lemma 11. $X, t \Vdash \phi \vee \psi$ iff $X, p \Vdash \phi$ or $X, p \Vdash \psi$ for all $t \leq p \in P_{X}$.

Proof. By fiddling. See the corollary to Theorem 1 in [7] for a proof sketch. 
Theorem 1 (Soundness). If $\phi \in \mathrm{RWQ}$, then hyp $\Vdash \phi$.

Proof. By straightforward induction on the proof of $\phi$.

\section{Correspondence Between Stratified Models and hyperdoctrines}

We will now provide a construction that takes a stratified model $\mathbf{T}$ and produces an 'equivalent' RW hyperdoctrine hyp ${ }^{\mathbf{T}}$. We first introduce a convenient bit of notation: if $\bar{x}=x_{1} x_{2} \ldots x_{n}$ and $\bar{y}=y_{1} y_{2} \ldots y_{n}$, then

$$
\bar{x}[t]_{X}^{\bar{y}}={ }^{x_{n}}\left[\cdots\left[{ }^{x_{2}}\left[{ }^{x_{1}}[t]_{X}^{y_{1}}\right]_{X}^{y_{2}}\right]_{X}\right]_{X}^{y_{n}}
$$

We will also write $\overline{n \times x}$ for the sequence containing $n x$ 's. To see this notation in action, we prove a few lemmas:

Lemma 12. If $\left|\overline{x_{1}}\right| \cap(Y-X)=\emptyset$ and $\left|\overline{x_{2}}\right| \cap(Y-X)=\emptyset$, then ${ }^{\overline{x_{1}}}\left[t \uparrow_{X}^{Y}\right]_{Y}^{\overline{x_{2}}}={ }^{\overline{x_{1}}}[t]_{X}^{\overline{x_{1}}} \uparrow_{X}^{Y}$

Proof. By repeated application of SM17.

Lemma 13. If $\left|\overline{y_{1}}\right| \cap X=\emptyset$ and $\left|\overline{y_{2}}\right| \cap X=\emptyset$, then ${ }^{\overline{y_{1}}}\left[t \uparrow_{X}^{Y}\right]_{Y}^{\overline{y_{2}}} \leq t \uparrow_{X}^{Y}$

Proof. By repeated application of the fact that ${ }^{a}[-]_{Y}^{b}$ is a closure operator.

Lemma 14. If $|\bar{x}| \subseteq X$ and $|\bar{y}| \subseteq Y-X$, then ${ }^{\bar{x}}\left[t \uparrow_{X}^{Y}\right]_{Y}^{\bar{y}} \downarrow_{X}^{Y} \leq t$

Proof. By repeated application of SM18.

Definition 9. Let $\mathbf{T}$ be a stratified model. Then we define $\mathbf{h y p}^{\mathbf{T}}$ as follows:

- If $\mathbf{T}(X)=\left\langle T_{X},{ }_{X}, \ell_{X}, \leq_{X}, P_{X}, *_{X}, \operatorname{Dom}_{X}, \operatorname{Dom}, I_{X}\right\rangle$, then $\operatorname{hyp}^{\mathbf{T}}(X)=\left\langle T_{X},{ }_{X}, \ell_{X}, \leq_{X}\right.$ $\left., P_{X}, *_{X}\right\rangle$.

- Let $X=\left|\overline{x_{1}}\right| \cup\left|\overline{x_{2}}\right| \cup \cdots \cup\left|\overline{x_{n}}\right|$ and suppose further that the $\left|\overline{x_{i}}\right|$ are the $\sim_{f^{-}}$ equivalence classes. (We will show below that which sequences $\overline{x_{i}}$ we choose is irrelevant). Finally, suppose that for each $i, x_{i}^{1}$ is the first member of $x_{i}$ and that $\overline{x_{i}}$ has length $l_{i}$. Then $\mathbf{h y p}_{f}^{\mathbf{T}}(t)=\overline{\overline{l_{1} \times x_{1}^{1}}} \overline{l_{n} \times x_{n}^{1}}\left[t \uparrow_{X}^{Y}\right]_{Y}^{\overline{x_{1}} \ldots \overline{x_{n}}}$. Since this is still quite cumbersome, we will write this as $\overline{\overline{\bar{l}_{i} \times x_{i}^{1}}}\left[t \uparrow_{X}^{Y}\right]_{Y}^{\overline{\bar{x}_{i}}}$.

Note that in the case where $f$ is the inclusion $X \subseteq Y$, $\operatorname{hyp}_{f}^{\mathbf{T}}(t)={ }^{\bar{x}}\left[t \uparrow_{X}^{Y}\right]_{Y}^{\bar{x}}=t \uparrow_{X}^{Y}$.

- $\Pi_{X \subseteq Y}=\downarrow_{Y}^{X}$ (we show below that $\downarrow_{Y}^{X}$ actually is right adjoint to $\uparrow_{Y}^{X}$ ).

I claim that hyp $\mathbf{p}^{\mathbf{T}}$, so defined, is an RW hyperdoctrine. I also claim that there is an assignment $\alpha^{\mathbf{T}}$ that we can associate to $\mathbf{T}$ in a natural way such that $\mathbf{T}, X, t \vDash \phi$ iff $\mathbf{h y p}^{\mathbf{T}}, X, t, \alpha^{\mathbf{T}} \Vdash \phi$. Before we can prove either of these claims, we first need to demonstrate that $\mathbf{h y p}^{\mathbf{T}}$ is well-defined. 
Lemma 15. If $|\bar{a}|=|\bar{b}|$ and $|\bar{c}|=|\bar{d}|$, then ${ }^{\bar{a}}[t]_{X}^{\bar{c}}={ }^{\bar{b}}[t]_{X}^{\bar{d}}$.

Proof Sketch: Use brute force to show that ${ }^{a_{2}}\left[{ }^{a_{1}}[t]_{X}^{b_{1}}\right]_{X}^{b_{2}}={ }^{b_{1}}\left[{ }^{a_{2}}[t]_{X}^{b_{2}}\right]_{X}^{b_{1}}$, then note that the result follows from this.

Lemma 16. Let $X$ be a finite set of variables and $C$ a subset of $X$. Let $|\bar{c}|=C$ and $|\bar{d}|=C$. Finally, suppose $\bar{c}$ has length $n$ and $\bar{d}$ has length $m$. Then ${ }^{\overline{n \times c_{1}}}[t]_{X}^{\bar{c}}=$ $\overline{m \times d_{1}}[t]_{X}^{\bar{d}}$.

Proof Sketch: We prove the result in a simple case that demonstrates the general principle. Let $C=\left\{x_{1}, x_{2}, x_{3}\right\} ; \bar{c}=x_{1}, x_{2}, x_{3}$, and $\bar{d}=x_{2}, x_{2}, x_{1}, x_{3}$. What we need to show is that

$$
x_{1}, x_{1}, x_{1}[t]_{X}^{x_{1}, x_{2}, x_{3}}={ }^{x_{2}, x_{2}, x_{2}, x_{2}}[t]_{X}^{x_{2}, x_{2}, x_{1}, x_{3}}
$$

To begin, note that by SM2,${ }^{x_{1}}[t]_{X}^{x_{1}} \leq t$, but also since ${ }^{x_{1}}[-]_{X}^{x_{1}}$ is a closure operator, $t \leq$ ${ }^{x_{1}}[t]_{X}^{x_{1}}$. Thus ${ }^{x_{1}}[-]_{X}^{x_{1}}$ is the identity map. So it will suffice to show that ${ }^{x_{1}, x_{1}}[t]_{X}^{x_{2}, x_{3}}=$ $x_{2}, x_{2}[t]_{X}^{x_{1}, x_{3}}$.

By SM2 and the fact that the various ${ }^{-}[-]_{-}^{-}$-operators are closure operators, $x_{1}, x_{1}[t]_{X}^{x_{2}, x_{3}}$ is symmetric both in $x_{1}$ and $x_{2}$ and in $x_{1}$ and $x_{3}$. So by the definition of 'symmetric', ${ }^{x_{1}, x_{1}}[t]_{X}^{x_{2}, x_{3}}$ is symmetric in $x_{2}$ and $x_{1}$ and in $x_{2}$ and $x_{3}$. By a similar argument we see that ${ }^{x_{2}, x_{2}}[t]_{X}^{x_{1}, x_{3}}$ is symmetric both in $x_{1}$ and $x_{2}$ and in $x_{1}$ and $x_{3}$.

Thus by SM2, ${ }^{x_{1}, x_{1}, x_{2}, x_{2}}[t]_{X}^{x_{2}, x_{3}, x_{1}, x_{3}}={ }^{x_{1}, x_{1}}[t]_{X}^{x_{2}, x_{3}}$ and ${ }^{x_{2}, x_{2}, x_{1}, x_{1}}[t]_{X}^{x_{1}, x_{3}, x_{2}, x_{3}}={ }^{x_{2}, x_{2}}[t]_{X}^{x_{1}, x_{3}}$. But by the previous lemma, ${ }^{X}, x_{1}, x_{2}, x_{2}[t]_{X}^{x_{2}, x_{3}, x_{1}, x_{3}}={ }^{x_{2}, x_{2}, x_{1}, x_{1}}[t]_{X}^{x_{1}, x_{3}, x_{2}, x_{3}}$, so ${ }^{x_{1}, x_{1}}[t]_{X}^{x_{2}, x_{3}}=$ $x_{2}, x_{2}[t]_{X}^{x_{1}, x_{3}}$.

Lemma 17. hyp $_{f}^{\mathbf{T}}$ is well-defined.

Proof. By the previous two lemmas, neither the choice of representing sequence $\overline{x_{i}}$ for the equivalence class $\left|\overline{x_{i}}\right|$ nor the order of the equivalence classes matters.

Lemma 18. If ${ }^{\bar{x}}[t]_{X}^{\bar{y}} \leq p \in P_{X}$, then for some $t \leq s,{ }^{\bar{x}}[s]_{X}^{\bar{y}} \leq p$ and ${ }^{\bar{x}}[s]_{X}^{\bar{y}} \in P_{X}$.

Proof. If $\bar{x}$ and $\bar{y}$ have length 1, the result follows from SM5. So suppose $\bar{x}=\widetilde{x} x_{n}$ and $\bar{y}=\widetilde{y} y_{n}$ for some $n \geq 2$. Then ${ }^{\bar{x}}[t]_{X}^{\bar{y}}={ }^{x_{n}}\left[{ }^{\widetilde{x}}[t]_{X}^{\tilde{y}}\right]_{X}^{y_{n}} \leq p \in P_{X}$. By SM5, for some ${ }^{\widetilde{x}}[t]_{X}^{\widetilde{y}} \leq$ $s,{ }^{x_{n}}[s]_{X}^{y_{n}} \leq p$, and ${ }^{x_{n}}[s]_{X}^{y_{n}} \in P_{X}$. First note that since $t \leq{ }^{\widetilde{x}}[t]_{X}^{\widetilde{y}}, t \leq s$ as well. Next notice that since ${ }^{\widetilde{x}}[t]_{X}^{\tilde{y}} \leq s, s$ is symmetric in $\widetilde{x}$ and $\widetilde{y}$. Thus since $s \leq{ }^{x_{n}}[s]_{X}^{y_{n}},{ }^{x_{n}}[s]_{X}^{y_{n}}$ is symmetric in $\widetilde{x}$ and $\widetilde{y}$ as well. So ${ }^{\widetilde{x}}\left[{ }^{x_{n}}[s]_{X}^{y_{n}}\right]_{X}^{\widetilde{y}}={ }^{x_{n}}[s]_{X}^{y_{n}}$. But also by Lemma 15, ${ }^{\widetilde{x}}\left[{ }^{x_{n}}[s]_{X}^{y_{n}}\right]_{X}^{\tilde{y}}={ }^{x_{n}}\left[{ }^{\widetilde{x}}[s]_{X}^{\tilde{y}}\right]{ }_{X}^{y_{n}}={ }^{\bar{x}}[s]_{X}^{\bar{y}}$. Thus ${ }^{\bar{x}}[s]_{X}^{\bar{y}} \leq p$ and ${ }^{\bar{x}}[s]_{X}^{\bar{y}} \in P_{X}$, as required.

Lemma 19. If ${ }^{\bar{x}}\left[t \uparrow_{X}^{Y}\right]_{Y}^{\bar{y}} \leq p$, then ${ }^{\bar{x}}\left[t \uparrow_{X}^{Y}\right]_{Y}^{\bar{y}} \leq{ }^{\bar{x}}\left[q \uparrow_{X}^{Y}\right]_{Y}^{\bar{y}} \leq p \in P_{Y}$ for some $t \leq q \in P_{X}$.

Proof. By the previous lemma, for some $t \uparrow_{X}^{Y} \leq s,{ }^{\bar{x}}[s]_{Y}^{\bar{y}} \leq p$ and ${ }^{\bar{x}}[s]_{Y}^{\bar{y}} \in P_{Y}$. I claim ${ }^{\bar{x}}[s]_{Y}^{\bar{y}} \downarrow_{X}^{Y}$ (which is in $P_{X}$ by SM16) does the job. To see this, first note that since $t \uparrow_{X}^{Y} \leq s \leq{ }^{\bar{x}}[s]_{Y}^{\bar{y}}$, we have that $t=t \uparrow_{X}^{Y} \downarrow_{X}^{Y} \leq{ }^{\bar{x}}[s]_{Y}^{\bar{y}} \downarrow_{X}^{Y}$. But then also $t \uparrow_{X}^{Y} \leq{ }^{\bar{x}}[s]_{Y}^{\bar{y}} \downarrow_{X}^{Y} \uparrow_{X}^{Y}$, so $\left.{ }^{\bar{x}}\left[t \uparrow_{X}^{Y}\right]_{Y}^{\bar{y}} \leq{ }^{\bar{x}}[s]_{Y}^{\bar{y}} \downarrow_{X}^{\bar{x}_{X}} \uparrow_{X}^{Y}\right]_{Y}^{\bar{y}}$. Finally note that $\left.{ }^{\bar{x}}\left[\bar{x}^{\bar{x}}[s]_{Y}^{\bar{y}} \downarrow_{X}^{Y_{X}} \uparrow_{X}^{Y}\right]_{Y}^{\bar{y}} \leq{ }^{\bar{x}}[s]_{Y}^{\bar{y}}\right]_{Y}^{\bar{y}}={ }^{\bar{x}}[s]_{Y}^{\bar{y}} \leq p$. 
Lemma 20. $\operatorname{hyp}_{f \circ g}^{\mathbf{T}}(t)=\operatorname{hyp}_{f}^{\mathbf{T}}\left(\mathbf{h y p}_{g}^{\mathbf{T}}(t)\right)$

Proof Sketch: Going through all the details is a notational nightmare, so we will only give the general idea, which is this: if we can show that $\mathbf{h y p}_{f}^{\mathbf{T}}\left(\mathbf{h y p}_{g}^{\mathbf{T}}(t)\right)$ is symmetric in $z_{1}$ and $z_{2}$ whenever $z_{1} \sim \sim_{f \circ g} z_{2}$, then using tricks from the proofs of previous, similar lemmas, we can show that $\mathbf{h y p} \mathbf{p}_{f \circ g}^{\mathbf{T}}(t) \leq \mathbf{h y p} \mathbf{p}_{f}^{\mathbf{T}}\left(\mathbf{h y p} \mathbf{p}_{g}^{\mathbf{T}}(t)\right)$. If we can show that $\mathbf{h y p}_{f \circ g}^{\mathbf{T}}(t)$ is symmetric in $z_{1}$ and $z_{2}$ whenever $z_{1} \sim_{f} z_{2}$ or $z_{1} \sim_{g} z_{2}$, then we can show that $\mathbf{h y p} \mathbf{p}_{f}^{\mathbf{T}}\left(\mathbf{h y p} \mathbf{p}_{g}^{\mathbf{T}}(t)\right) \leq \mathbf{h y} \mathbf{p}_{f \circ g}^{\mathbf{T}}(t)$. But both of these follow easily from the definition of $\sim_{f \circ g}$, proving the lemma.

Theorem 2. If $\mathbf{T}$ is a stratified model, then $\mathbf{h y p}^{\mathbf{T}}$ is an RW hyperdoctrine.

Proof. We must verify that hyp ${ }^{\mathbf{T}}$ satisfies RH1-RH6. For RH1, we need to show that hyp $^{\mathbf{T}}$ is a functor whose image is in Frame. The only difficult parts are showing that $\mathbf{h y p}_{f}^{\mathbf{T}}$ is a frame morphism (but this follows from monotonicity for $\uparrow$, SM4, the fact that ${ }^{a}[t]_{X}^{b}$ is a closure operator, and Lemma 19) and that $\mathbf{h y p}_{f \circ g}^{\mathbf{T}}=\mathbf{h y p}_{f}^{\mathbf{T}} \circ \mathbf{h y} \mathbf{p}_{g}^{\mathbf{T}}$. The latter follows from Lemma 20

For RH2 we need to show that for each $f: X \longrightarrow X$,

(a) If $t \in \operatorname{hyp}^{\mathbf{T}}(X)$ then $t \leq \mathbf{h y p}_{f}^{\mathbf{T}}(t)$;

(b) If $p \in P_{X}$, then $\mathbf{h y p}_{f}^{\mathbf{T}}(p) \in P_{X}$;

(c) $\operatorname{hyp}_{f}^{\mathbf{T}}\left(t \mathbf{h y p}_{f}^{\mathbf{T}}(u)\right)=\mathbf{h y p}_{f}^{\mathbf{T}}(t) u$; and

(d) $\mathbf{h y p}_{f}^{\mathbf{T}}\left(\mathbf{h y p}_{f}^{\mathbf{T}}(p)^{*}\right)=\mathbf{h y p}_{f}^{\mathbf{T}}(p)^{*}$.

(a) follows from the fact that each ${ }^{a}[-]_{X}^{b}$ is a closure operator. (b) and (d) follow from SM3. For (c), first notice that by SM $4,{ }^{a}\left[t^{a}[u]_{X}^{b}\right]_{X}^{b} \leq{ }^{a}[t]_{X}^{b} u$. For the converse inequality, we argue as follows:

$$
\begin{array}{rlr}
{ }^{a}[t]_{X}^{b} u & \leq{ }^{a}[t]_{X}^{b} a[u]_{X}^{b} & \text { because } u \leq{ }^{a}[u]_{X}^{b} \\
& ={ }^{a}[u]_{X}^{b}{ }^{a}[t]_{X}^{b} & \text { by commutativity } \\
& \leq{ }^{a}[u]_{X}^{b} t & \text { by SM4 } \\
& =t^{a}[u]_{X}^{b} & \text { by commutativity } \\
& \leq{ }^{a}\left[t^{a}[u]_{X}^{b}\right]_{X}^{b} & \text { since }^{a}[-]_{X}^{b} \text { is a closure operator }
\end{array}
$$

Thus ${ }^{a}\left[t^{a}[u]_{X}^{b}\right]_{X}^{b}={ }^{a}[t]_{X}^{b} u$.

For RH3, we need that $\uparrow_{X}^{Y}$ is exact and that $\downarrow_{X}^{Y}$ is right adjoint to $\uparrow_{X}^{Y}$. Exactness follows from SM11. For adjointness, it suffices to show that $s \uparrow_{X}^{Y} \leq t$ iff $s \leq t \downarrow_{X}^{Y}$. So, suppose $s \uparrow_{X}^{Y} \leq t$. Then by SM8 $s \uparrow_{X}^{Y} \downarrow_{X}^{Y}=s \leq t \downarrow_{X}^{Y}$. Now suppose that $s \leq t \downarrow_{X}^{Y}$. Then by SM10 $s \uparrow_{X}^{Y} \leq t \downarrow_{X}^{Y} \uparrow_{X}^{Y} \leq t$.

For RH4 we need that $\mathbf{h y p}_{f}^{\mathbf{T}}(t) \downarrow_{X}^{Y} \leq \mathbf{h y p}_{f \mid X}^{\mathbf{T}}(t)$ (which we deal with below); that $P_{Y} \downarrow_{X}^{Y} \subseteq P_{X}$; and that $\left(p^{*}\right) \downarrow_{X}^{Y}=\left(p \downarrow_{X}^{Y}\right)^{*}$. But both of these follow from SM16, so we have only the first matter to address.

Say that the pair $\langle w, z\rangle$ is of 
- Type 1 if both $w$ and $z$ are in $X$;

- Type 2 if both $w$ and $z$ are in $Y-X$.

- Type 3 if $w \in X$ but $z \in Y-X$; and

By Lemma 15, we can rewrite $\overline{\overline{\bar{l}_{i} \times x_{i}}}\left[t \uparrow_{X}^{Y}\right]_{Y}^{\overline{\bar{x}_{i}}}$ in the form $\overline{\overline{\overline{w^{1}}}} \overline{\overline{w^{2}}} \overline{\overline{w^{3}}}\left[t \uparrow_{X}^{Y}\right] \overline{\overline{z^{1}}} \overline{\overline{z^{2}}} \overline{\overline{z^{3}}}$ where each pair $\left\langle w_{i}^{1}, z_{i}^{1}\right\rangle$ is type 1 , each pair $\left\langle w_{i}^{2}, z_{i}^{2}\right\rangle$ is type 2 , and each pair $\left\langle w_{i}^{3}, z_{i}^{3}\right\rangle$ is type 3 . We then see that

$$
\begin{aligned}
& \mathbf{h y p}_{f}^{\mathbf{T}}(t) \downarrow_{X}^{Y}=\overline{\overline{w^{1}}} \overline{\overline{w^{2}}} \overline{\overline{w^{3}}}\left[t \uparrow_{X}^{Y}\right] \overline{\overline{z_{Y}^{1}}} \overline{\overline{z^{2}}} \overline{\overline{z^{3}}} \downarrow_{X}^{Y}
\end{aligned}
$$

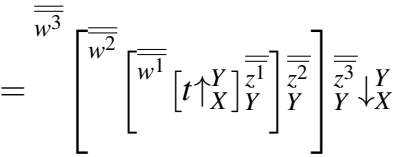

$$
\begin{aligned}
& \left.=\sqrt[\overline{\overline{w^{3}}}]{\overline{\overline{w^{2}}}}\left[\overline{\overline{w^{1}}}[t]_{X}^{\overline{z^{1}}} \uparrow_{X}^{Y}\right] \overline{\overline{z^{2}}}\right] \overline{\overline{z^{3}}} \downarrow_{Y}^{Y} \quad \text { by Lemma } 12 \\
& \leq \overline{\overline{w^{3}}}\left[\overline{\overline{w^{1}}}[t]_{X}^{\overline{\bar{z}}} \uparrow_{X}^{Y}\right]_{Y}^{\overline{\overline{z^{3}}}} \downarrow_{X}^{Y} \quad \text { by Lemma } 13 \\
& \leq \leq^{\overline{\overline{w^{1}}}}[t]_{X}^{\overline{\overline{\bar{q}^{1}}}} \quad \text { by Lemma } 14 \\
& =\operatorname{hyp}_{\left.f\right|_{X}}^{\mathbf{T}}(t)
\end{aligned}
$$

For RH5 we need to show that if $Y \subseteq Z$ and $X \cap Z=\emptyset$, the following commutes:

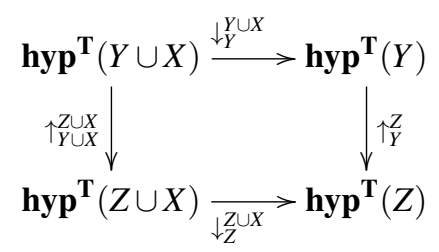

But since $X \cap Z=\emptyset$, it follows that $Z \cap(Y \cup X)=Z \cap Y=Y$. So by SM9, $t \downarrow_{Y}^{Y \cup X} \uparrow_{Y}^{Z}=t \uparrow \uparrow_{Y \cup X}^{Z} \downarrow_{Z} \cup X X$

Finally, for RH6, we need to show that if $s \in \operatorname{hyp}^{\mathbf{T}}(X)$ and $t \in \operatorname{hyp}^{\mathbf{T}}(Y)$, and $X \subseteq Y$, then $\left(s \uparrow_{X}^{Y} t\right) \downarrow_{X}^{Y} \leq s t \downarrow_{\downarrow}^{Y}$. But after a bit of commutativity, this is exactly the content of SM12.

Definition 10. If $\mathbf{T}$ is a stratified model, then $\alpha^{\mathbf{T}}(X, R \bar{x})=\left\{s: I_{X}(\bar{x}) \in I(s)(R)\right\}$.

Lemma 21. $\alpha^{\mathbf{T}}$ is an assignment for hyp $\mathbf{p}^{\mathbf{T}}$

Proof. We need to verify that $\alpha^{\mathbf{T}}$ meets conditions A1-A4 from Definition 8. A1, $\mathrm{A} 2$, and A4 are straightforward and left to the reader. For A3, let $f: X \longrightarrow Y$. $\mathbf{h y p}_{f}^{\mathbf{T}}(t) \in \alpha^{\mathbf{T}}(Y, R \bar{y})$ iff $I(\bar{y}) \in I_{Y}\left(\mathbf{h y p}_{f}^{\mathbf{T}}(t)\right)(R)$. Let $\bar{z} \sim_{f} \bar{y}$. By the definition of 
$\mathbf{h y p}_{f}^{\mathbf{T}}(t)$, it is symmetric in $z_{1}$ and $y_{1}$ and in $z_{2}$ and $y_{2}$, etc. Thus if $\mathbf{h y p}_{f}^{\mathbf{T}}(t) \in$ $\alpha^{\mathbf{T}}(Y, R \bar{y})$ then $I(\bar{z}) \in I_{Y}\left(\mathbf{h y p} \mathbf{p}_{f}^{\mathbf{T}}(t)\right)(R)$ for all $\bar{z} \sim_{f} \bar{y}$, which is to say that $\mathbf{h y p}_{f}^{\mathbf{T}}(t) \in$ $\alpha^{\mathbf{T}}(Y, R \bar{z})$ for all $\bar{z} \sim_{f} \bar{y}$. The converse is trivial.

Theorem 3. For all $\phi$, $\mathbf{h y p}^{\mathbf{T}}, X, t, \alpha^{\mathbf{T}} \Vdash \phi$ iff $\mathbf{T}, X, t \vDash \phi$

Proof. By a straightforward induction on $\phi$.

From here, completeness for RW hyperdoctrines is only a short step away:

Corollary 1. If hyp $\Vdash \phi$ then $\phi \in \mathrm{RWQ}$.

Proof. Suppose $\phi \notin \mathrm{RWQ}$. Then there is a stratified model $\mathbf{T}$ so that $\mathbf{T}, X, t \not \not \phi$ for some $X$ and some $t \in \mathbf{T}(X)$. But then $\mathbf{h y p}^{\mathbf{T}}, X, t, \alpha^{\mathbf{T}} \forall \phi$. So hyp $\forall \phi$.

\section{Ontology}

Recall from the introduction that the standard interpretation of the base category C of a hyperdoctrine is one of the few constants in the otherwise-unsettled hyperdoctrine literature: its objects are types and its arrows are terms. Also recall that I presented RW hyperdoctrines as a type of covariant functor, but the usual ontological interpretation of the base category of a hyperdoctrine is given with respect to contravariant functors. But nothing could be easier to fix: a covariant functor EqRIn $\longrightarrow$ Frame is exactly the same thing as a contravariant functor EqRIn ${ }^{\text {op }} \longrightarrow$ Frame. Thus the 'type theory' associated to an RW hyperdoctrine is given by the category EqRIn ${ }^{\text {op }}$.

The problem is that this category seems to reflect no coherent ontology at all. It certainly shows no ontology of arbitrary objects. Expanding on this a bit, the objects of EqRIn are finite sets of variables. Thus, each singleton set $\left\{v_{i}\right\}$ represents a different type, as does each doubleton set $\left\{v_{i}, v_{j}\right\}$, etc. But it's not at all clear why we should regard distinct variables as distinct types - typically, one would expect all the variables to be of the same type. What's worse is that these types aren't related to one another in nice ways. For example, singleton types are neither initial nor terminal (in EqRIn, there are two arrows $\left\{v_{i}\right\} \longrightarrow\left\{v_{i}, v_{j}\right\}$ and none in the other direction). And doubleton types are neither products nor sums of their singleton retractions.

This could perhaps be mollified if we could think of distinct variables as distinct arbitrary objects. But there's no clear way to do this. One would expect that if an ontology of arbitrary objects were to show itself somewhere, it should show itself by presenting terms - which is to say arrows of $\mathbf{E q R} \mathbf{R n}^{\text {op }}$ - corresponding to different arbitrary objects. But looking at the simplest case seems to throw water on this way of reading the terms in EqRIn ${ }^{\text {op }}$. For example, there are two arrows in EqRIn ${ }^{\text {op }}$ from $\left\{v_{1}, v_{2}\right\}$ to $\left\{v_{1}\right\}$. Focusing only on the equivalence relations, they are

- $v_{1} \sim_{1} v_{1} ; v_{2} \sim_{1} v_{2} ;$ and 
- $v_{1} \sim_{2} v_{1} ; v_{2} \sim_{2} v_{2} ; v_{1} \sim_{2} v_{2} ; v_{2} \sim_{2} v_{1}$.

Perhaps we could squint and see $\sim_{2}$ as a way of regarding $v_{1}$ as standing arbitrarily for $v_{1}$ or for $v_{2}$. But I see no obvious interpretation of $\sim_{1}$ at all.

Of course, all this leaves open the possibility that perhaps the repackaging I present here is, despite being the natural option, not the correct one. Or perhaps there's some way to interpret EqRIn ${ }^{\text {op }}$ - despite all appearances - as a category of types and terms. But we will have to leave investigation of these questions to another time.

Fine, to an extent, acknowledges problems in this neighborhood while introducing stratified semantics. Here is his discussion of the matter:

In the earlier work, [author's note: this seems to be a reference to $[11,8]$ ] there exists an explanation of the truth of statements about arbitrary objects in terms of individuals; a statement about an arbitrary object is true just in case it is true for all individuals. On the present account, there is no such explanation. Truths about arbitrary objects are just given; they are, in my previous terminology, about those objects as objects in their own right. Another difference is that the notion of dependence is integral to my earlier work, but in the present account does not even make an appearance.[9, p. 30]

So the situation the relevance logician finds herself in is this: she has available a technically adequate semantic theory in stratified semantics. There is one philosophical interpretation of this theory that has been - albeit tentatively - put forward. It involves arbitrary objects. But on closer inspection, it doesn't seem it can be sustained.

The extent to which this is a problem depends on how serious a role you think ontological considerations should play in your choice of logic. But if you think they should play any serious role, it seems your left saying that stratified semantics is, at best, on shaky footing.

\section{Appendix A: Soundness for Stratified Models}

Lemma 22 (HH - Horizontal Heredity). If $X, s \vDash \phi$ and $s \leq t$, then $X, t \vDash \phi$.

Proof. By a straightforward induction on $\phi$.

Lemma 23. $t \downarrow_{X}^{Y} u \downarrow_{X}^{Y} \leq(t u) \downarrow_{X}^{Y}$.

Proof. By fiddling with arrows; the discussion preceding Lemma 1 in [9] has hints.

Lemma 24 (Symmetry). $X,{ }^{a}[t]_{X}^{b} \vDash \phi(a)$ iff $X,{ }^{a}[t]_{X}^{b} \vDash \phi(b)$.

Proof. By induction on $\phi$. The base case follows from SM2; and the conjunction case is trivial. In each of the remaining cases, the 'if' direction is the same as that 'only if' direction, so we deal only with the former.

For the negation case, let $X,{ }^{a}[t]_{X}^{b} \mid \forall \neg \psi(b)$. Then $X, p^{*} \vDash \psi(b)$ for some ${ }^{a}[t]_{X}^{b} \leq$ $p \in P_{X}$. By SM5, for some $u$ with ${ }^{a}[u]_{X}^{b} \in P_{X},{ }^{a}[t]_{X}^{b} \leq{ }^{a}[u]_{X}^{b} \leq p$. Thus $p^{*} \leq\left({ }^{a}[u]_{X}^{b}\right)^{*}$. 
So by HH, $X,\left({ }^{a}[u]_{X}^{b}\right)^{*} \vDash \psi(b)$. But by SM3, ${ }^{a}\left[\left({ }^{a}[u]_{X}^{b}\right)^{*}\right]_{X}^{b}=\left({ }^{a}[u]_{X}^{b}\right)^{*}$. Thus by IH, $X,\left({ }^{a}[u]_{X}^{b}\right)^{*} \vDash \psi(a)$. So $X,{ }^{a}[t]_{X}^{b} \forall \neg \psi(a)$.

For conditionals, first suppose $X,{ }^{a}[t]_{X}^{b} \vDash \phi_{1}(a) \rightarrow \phi_{2}$. Let $X, u \vDash \phi_{1}(b)$. Then by $\mathrm{IH}, X,{ }^{a}[u]_{X}^{b} \vDash \phi_{1}(a)$. Thus $X,{ }^{a}[t]_{X}^{b}{ }^{a}[u]_{X}^{b} \vDash \phi_{2}$. By SM4,${ }^{a}[t]_{X}^{b}{ }^{a}[u]_{X}^{b} \leq{ }^{a}[t]_{X}^{b} u$. Thus by $\mathrm{HH}, X,{ }^{a}[t]_{X}^{b} u \vDash \phi_{2}$ as well. So $X,{ }^{a}[t]_{X}^{b} \vDash \phi_{1}(b) \rightarrow \phi_{2}$.

Suppose instead that $X,{ }^{a}[t]_{X}^{b} \vDash \phi_{1} \rightarrow \phi_{2}(a)$. Let $X, u \vDash \phi_{1}$. Then $X,{ }^{a}[t]_{X}^{b} u \vDash \phi_{2}(a)$. Thus, by IH, $X,{ }^{a}\left[{ }^{a}[t]_{X}^{b} u\right]_{X}^{b} \vDash \phi_{2}(b)$. But by SM4 and the fact that ${ }^{a}[-]_{X}^{b}$ is a closure operator, ${ }^{a}\left[{ }^{a}[t]_{X}^{b} u\right]_{X}^{b} \leq{ }^{a}[t]_{X}^{b} u$. So by HH $X,{ }^{a}[t]_{X}^{b} u \vDash \phi_{2}(b)$. So $X,{ }^{a}[t]_{X}^{b} \vDash \phi_{1} \rightarrow \phi_{2}(b)$.

Finally we deal with universals. Suppose $X,{ }^{a}[t]_{X}^{b} \vDash \forall z \psi(z, a)$. Then $Y,{ }^{a}[t]_{X}^{b} \uparrow_{X}^{Y} \vDash$ $\psi(y, a)$ for some $Y \supsetneq X$ and $y \in Y-X$. Thus by SM17, $Y,{ }^{a}\left[t \uparrow_{X}^{Y}\right]_{Y}^{b} \vDash \psi(y, a)$. The inductive hypothesis then gives that $Y,{ }^{a}\left[t \uparrow_{X}^{Y}\right]_{Y}^{b} \vDash \psi(y, b)$. So by SM17 again, $Y,{ }^{a}[t]_{X}^{b} \uparrow_{X}^{Y} \vDash$ $\psi(y, b)$. Thus $X,{ }^{a}[t]_{X}^{b} \vDash \forall z \psi(z, b)$.

Lemma 25 (DH - Downward Heredity). If $X \subseteq Y$, and $\phi \in \mathscr{L}_{X}$ then $Y, t \vDash \phi$ iff $X, t \downarrow_{X}^{Y} \vDash \phi$.

Proof. By induction on $\phi$. Most cases are straightforward. The universal case is not.

Suppose $Y, t \vDash \forall v \psi(v)$. Then $Z, t \uparrow_{Y}^{Z} \vDash \psi(z)$ for some $Z \supsetneq Y$ and $z \in Z-Y$. Let $W=X \cup(Z-Y)$. Notice that $Y \cap W=X$. So by SM9, $t \uparrow_{Y}^{Z} \downarrow_{W}^{Z}=t \downarrow_{X}^{Y} \uparrow_{X}^{W}$. But by IH, $W, t \uparrow_{Y}^{Z} \downarrow_{W}^{Z} \vDash \psi(z)$. So $W, t \downarrow_{X}^{Y} \uparrow_{X}^{W} \vDash \psi(z)$. Thus $X, t \downarrow_{X}^{Y} \vDash \forall v \psi(v)$.

Now suppose $X, t \downarrow_{X}^{Y} \vDash \forall k \psi(k)$. Then $Z, t \downarrow_{X}^{Y} \uparrow_{X}^{Z} \vDash \psi(z)$ for some $Z \supsetneq X$ and $z \in$ $Z-X$. At this point the proof splits into two cases.

Case 1: Suppose $z \notin Y$. Notice that $t \downarrow_{X}^{Y} \uparrow_{X}^{Z \cup Y} \downarrow_{Z}^{Z U Y}=t \downarrow_{X}^{Y} \uparrow_{X}^{Z} \uparrow_{Z}^{Z}{ }_{Z} \cup Y \downarrow_{Z}^{Z} \cup Y Y=t \downarrow_{X}^{Y} \uparrow_{X}^{Z}$. So since $Z, t \downarrow_{X}^{Y} \uparrow_{X}^{Z} \vDash \psi(z)$, IH gives that $Z \cup Y, t \downarrow_{X}^{Y} \uparrow_{X}^{Z} \cup Y=\psi(z)$ as well. But $t \downarrow_{X}^{Y} \uparrow_{X}^{Z \cup Y}=$ $t \downarrow_{X}^{Y} \uparrow_{X}^{Y} \uparrow_{Y}^{Y} \cup Z \leq t \uparrow_{Y} \cup \cup Y$. So by HH, $Z \cup Y, t \uparrow_{Y}^{Z \cup Y} \vDash \psi(z)$. Since $z \notin Y$, this shows that $Y, t \vDash \forall k \psi(k)$.

Case 2: suppose $z \in Y$. Choose $w \notin Z \cup Y$. Let $X^{+}=X \cup\{w\}, Y^{+}=Y \cup\{w\}$ and $Z^{+}=Z \cup\{w\}$. By a similar argument as before, $t \downarrow_{X}^{Y} \uparrow_{X}^{Z^{+}} \cup Y^{+} \downarrow_{Z}^{Z^{+} \cup Y^{+}}=t \downarrow_{X}^{Y} \uparrow_{X}^{Z}$. Since $Z, t \downarrow_{X}^{Y} \uparrow_{X}^{Z} \vDash \psi(z)$, by IH, $Z^{+} \cup Y^{+}, t \downarrow_{X}^{Y} \uparrow_{X}^{Z^{+} \cup Y^{+}} \vDash \psi(z)$. So since $t \downarrow_{X}^{Y} \uparrow_{X}^{Z^{+} \cup Y^{+}} \leq$ ${ }^{w}\left[t \downarrow_{\downarrow_{X}}^{Y} \uparrow_{X}^{Z^{+} \cup Y^{+}}\right]_{Z^{+} \cup Y^{+}}^{z}$, HH gives $Z^{+} \cup Y^{+},{ }^{w}\left[t \downarrow_{X}^{Y} \uparrow_{X}^{Z^{+} \cup Y^{+}}\right]_{Z^{+} \cup Y^{+}} \vDash \psi(z)$. Thus by symmetry $Z^{+} \cup Y^{+},{ }^{w}\left[t \downarrow_{X}^{Y} \uparrow_{X}^{Z^{+} \cup Y^{+}}\right]_{Z^{+} \cup Y^{+}} \vDash \psi(w)$. So since $w \in X^{+}$, IH then gives $X^{+},{ }^{w}\left[t \downarrow_{X}^{Y} \uparrow_{X}^{Z^{+} \cup Y^{+}}\right]_{Z^{+} \cup Y^{+}} \downarrow_{X^{+}}^{Z^{+} \cup Y^{+}} \vDash \psi(w)$.

Finally, notice that since $z \in\left(Z^{+} \cup Y^{+}\right)-X^{+}$, we have that

$$
\begin{aligned}
{ }^{z}\left[t \downarrow_{X}^{Y} \uparrow_{X}^{Z^{+} \cup Y^{+}}\right]_{Z^{+} \cup Y^{+}}^{w} \downarrow_{X^{+}}^{Z^{+} \cup Y^{+}} & \left.={ }^{z}\left[t \downarrow_{X}^{Y} \uparrow_{X}^{X^{+}} \uparrow_{X^{+}}^{Z^{+} \cup Y^{+}}\right]\right]_{Z^{+} \cup Y^{+}} \downarrow_{X^{+}}^{Z^{+} \cup Y^{+}} \\
& \leq t \downarrow_{X}^{Y} \uparrow_{X}^{X^{+}}
\end{aligned}
$$

Thus by HH, $X^{+}, t \downarrow_{X}^{Y} \uparrow_{X}^{X^{+}} \vDash \psi(w)$. But since $w \notin Y$, we now find ourselves (after swapping $X^{+}$for $Z$ and $w$ for $z$ ) in exactly the same situation as in the previous case. Thus by the argument given there, $Y, t \vDash \forall v \psi(v)$.

Corollary 2. If $Y, s \uparrow_{X}^{Y} \vDash \phi(y)$ for some $Y \supsetneq X$ and $y \in Y-X$, then $X, s \vDash \phi(x)$ for all $x \in X$. 
Proof. Let $x \in \operatorname{Dom}_{X}$. Since $s \uparrow_{X}^{Y} \leq{ }^{x}\left[s \uparrow_{X}^{Y}\right]_{Y}^{y}$, HH gives that $Y,{ }^{x}\left[s \uparrow_{X}^{Y}\right]_{Y}^{y} \vDash \phi(y)$. Thus by symmetry $Y,{ }^{x}\left[s \uparrow_{X}^{Y}\right]_{Y}^{y} \vDash \phi(x)$. So by DH, $Y,{ }^{x}\left[s \uparrow_{X}^{Y}\right]_{Y}^{y} \downarrow_{X}^{Y} \vDash \phi(x)$. Since $y \in Y-X$, SM18 gives that ${ }^{x}\left[s \uparrow_{X}^{Y}\right]_{Y}^{y} \downarrow_{X}^{Y} \leq s$. So by HH, $X, s \vDash \phi(x)$.

Theorem 4. If $\phi \in \mathrm{RWQ}$, then $\operatorname{Str} \vDash \phi$.

Proof. By a straightforward induction on the proof of $\phi$.

\section{Appendix B: Completeness}

We begin with some definitions:

- Write $\Sigma \succ \phi$ when there is a sequence $\phi_{1}, \phi_{2}, \ldots, \phi_{n}$ such that $\phi_{n}=\phi$ and for all $1 \leq i \leq n$, either

- $\phi_{i} \in \Sigma$; or

- For some $j<i$ and $k<i, \phi_{i}=\phi_{j} \wedge \phi_{k}$; or

- For some $j<i, \phi_{j} \rightarrow \phi_{i} \in \mathrm{RWQ}$.

- Write $\Sigma \succ^{k, l} \phi$ when there is a sequence $\phi_{1}, \phi_{2}, \ldots, \phi_{n}$ such that $\phi_{n}=\phi$ and for all $1 \leq i \leq n$, either

$-\phi_{i} \in \Sigma$; or

- For some $j<i$ and $k<i, \phi_{i}=\phi_{j} \wedge \phi_{k}$; or

- For some $j<i, \phi_{j} \rightarrow \phi_{i} \in \mathrm{RWQ}$; or

- For some $j<i, \phi_{i}=\phi_{j}(k / l)$; or

- For some $j<i, \phi_{i}=\phi_{j}(l / k)$.

- Write $\phi(x / / y)$ for the result of replacing every occurrence of $x$ in $\phi$ with $y$.

- Write $c(\Sigma)$ for $\{\phi: \Sigma \succ \phi\}$ and $c_{X}(\Sigma)$ for $\left\{\phi \in \mathscr{L}_{X}: \Sigma \succ \phi\right\}$.

- Write $c^{k, l}(\Sigma)$ for $\left\{\phi: \Sigma \succ^{k, l} \phi\right\}$ and write $c_{X}^{k, l}(\Sigma)$ for $\left\{\phi \in \mathscr{L}_{X}: \Sigma \succ^{k, l} \phi\right\}$.

- Say $\Sigma$ is an $X$-theory when $\Sigma \subseteq \mathscr{L}_{X}$ and for all $\phi \in \mathscr{L}_{X}, \Sigma \succ \phi$ only if $\phi \in \Sigma$.

- Say $\Sigma$ is an $X^{k, l}$-theory when $\Sigma \subseteq \mathscr{L}_{X}$ and for all $\phi \in \mathscr{L}_{X}, \Sigma \succ^{k, l} \phi$ only if $\phi \in \Sigma$.

- We write $\mathrm{RWQ}_{X}$ for RWQ $\cap \mathscr{L}_{X}$.

- Say $\Sigma$ is $X$-normal when $\mathrm{RWQ}_{X} \subseteq \Sigma$.

- Say $\Sigma$ is prime when $\alpha \vee \beta \in \Sigma$ only if $\alpha \in \Sigma$ or $\beta \in \Sigma$.

- Say $\Sigma$ is closed under disjunction when $\alpha \vee \beta \in \Sigma$ whenever $\alpha \in \Sigma$ and $\beta \in \Sigma$.

Lemma 26 (Deduction Lemma). If $\alpha \succ \beta$, then $\alpha \rightarrow \beta \in \mathrm{RWQ}$.

Lemma 27. If $\Sigma \succ \phi(k), k$ not free in $\Sigma$ but free for $x$ in $\phi(k)$, then $\Sigma \succ \forall x \phi(x)$.

Lemma 28 (Lindenbaum). Suppose $\Delta$ is closed under disjunction, $\Gamma$ is an $X$-(or

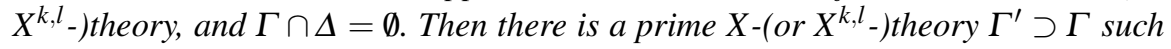
that $\Gamma^{\prime} \cap \Delta=\emptyset$.

Lemma 29. If $\Gamma$ is a theory and $\left\{\Gamma_{i}\right\}_{i \in I}$ is the set of all prime theories that contain $\Gamma$, then $\Gamma=\cap_{i \in I} \Gamma_{i}$ 
Lemma 30. For each $X, \mathrm{RWQ}_{X}$ is a prime $X$-theory.

For proofs of Lemmas 26-30, see [15]. We also need the following facts about RWQ:

Fact $1(\phi \rightarrow(\psi \rightarrow \rho)) \rightarrow(\psi \rightarrow(\phi \rightarrow \rho)) \in$ RWQ

Fact $2 \phi \rightarrow(\psi \rightarrow((\phi \rightarrow(\psi \rightarrow \rho)) \rightarrow \rho)) \in$ RWQ

Fact $3 \phi \rightarrow \neg \neg \phi \in$ RWQ.

Fact $4 \phi \rightarrow(\phi \vee \psi) \in$ RWQ.

Fact $5 \neg(\phi \wedge \psi) \rightarrow(\neg \phi \vee \neg \psi) \in$ RWQ.

Fact $6(\phi \rightarrow \psi) \rightarrow(\neg \psi \rightarrow \neg \phi) \in$ RWQ.

Finally, we will have need of the following two lemmas whose proofs we leave to the reader:

Lemma 31. $\rho \succ^{k, l} \psi$ iff RWQ $\succ^{k, l} \rho \rightarrow \psi$ iff $\rho(l / / k) \rightarrow \psi(l / / k) \in \mathrm{RWQ}$

Lemma 32. If $\mathrm{RWQ} \succ^{k, l} \rho \rightarrow \psi$ and $\Sigma \succ^{k, l} \psi \rightarrow \phi$, then $\Sigma \succ^{k, l} \rho \rightarrow \phi$.

Definition 11. For finite $X \subseteq$ Var we define $\mathbf{M}(X)$ as $\left\langle T_{X},{ }^{\cdot}, \mathrm{RWQ}_{X}, \subseteq, \Pi_{X}, *_{X}, X, I_{X}\right\rangle$, where $T_{X}$ is the set of $X$-theories, $t \cdot X u=\{\psi: \phi \rightarrow \psi \in t$ and $\phi \in u\}, \Pi_{X}$ is the set of prime $X$-theories, $t^{*}=\left\{\phi \in \mathscr{L}_{X}: \neg \phi \notin t\right\}$, and $I_{X}(t)(P)=\{\bar{x}: P \bar{x} \in t\}$.

Lemma 33. $\left\langle T_{X}, \cdot X\right\rangle$ is a commutative monoid for which $\mathrm{RWQ}_{X}$ serves as an identity element. Further, if $a$ and $b$ are in $\Pi_{X}$ and $t a \subseteq b$, then $t b^{* X} \subseteq a^{* X}$, and $\left(a^{* X}\right)^{* X}=a$.

Proof. By a straightforward application of the facts and definitions above.

Lemma 34. If $p \in \Pi_{X}$, then $p^{*} \in \Pi_{X}$.

Proof. We first prove that $p^{*}$ is an $X$-theory. So let $\phi \in \mathscr{L}_{X}$ and $p^{*_{X}} \succ \phi$. We show by induction on the length of the proof witnessing $p^{* X} \succ \phi$ that $\phi \in p^{* X}$. Let $\psi_{1}, \ldots, \psi_{i}=\phi$ be such a proof. If $i=1$, then $\psi_{1}=\phi \in p^{* X}$, so we are done. Now suppose $i>1$. Clearly if $\psi_{i} \in p^{* X}$ we are done.

Next suppose for some $j<i$ and $k<i, \phi=\psi_{j} \wedge \phi_{k}$. Then by IH, $\psi_{j} \in p^{* X}$ and $\psi_{k} \in p^{* X}$. So $\neg \psi_{j}$ and $\neg \psi_{k}$ are not in $p$. So since $p$ is prime, $\neg \psi_{j} \vee \neg \psi_{k}$ is not in $p$. Since $p$ is a theory, it follows by Fact 5 that $\neg\left(\psi_{j} \wedge \psi_{k}\right) \notin p$. So $\psi_{j} \wedge \psi_{k} \in p^{*_{X}}$ as required.

Finally, suppose for some $j<i, \psi_{j} \rightarrow \phi \in \mathrm{RWQ}$. Then by inductive hypothesis, $\psi_{j} \in p^{* X}$. So $\neg \psi_{j} \notin p$. Since $\psi_{j} \rightarrow \phi \in \mathrm{RWQ}$, Fact 6 gives that $\neg \phi \rightarrow \neg \psi_{j} \in \mathrm{RWQ}$. Thus since $p$ is a theory and $\neg \psi_{j} \notin p, \neg \phi \notin p$. Thus $\phi \in p^{* X}$ as required.

Now we show that $p^{* X}$ is prime. So, suppose $\phi \vee \psi \in p^{* X}$ but that neither $\phi$ nor $\psi$ is in $p^{* X}$. Then $\neg(\phi \vee \psi) \notin p$ but $\neg \phi \in p$ and $\neg \psi \in p$. Since $p$ is a theory, it follows that $\neg \phi \wedge \neg \psi \in p$. So by Fact $3, \neg \neg(\neg \phi \wedge \neg \psi) \in p$. But by the definition of ' $\vee$ ', $\neg \neg(\neg \phi \wedge \neg \psi) \in p$ gives that $\neg(\phi \vee \psi) \in p$, contradicting $\neg(\phi \vee \psi) \notin p$. Thus, either $\phi \in p^{* X}$ or $\psi \in p^{* X}$, so $p^{* X}$ is prime.

Lemma 35. For all $a$ and $b$ in $T_{X}$, if $p \in \Pi_{X}$ and $a b \subseteq p$ then there are $q_{1}$ and $q_{2}$ in $\Pi_{X}$ so that $a \subseteq q_{1}$ and $q_{1} b \subseteq p$, and $b \subseteq q_{2}$ and $a q_{2} \subseteq p$. 
Proof Sketch. The result follows from several straightforward applications of the Lindenbaum lemma, so I leave some of the details to the reader. To begin, let $\theta_{1}=$ $\left\{\phi: c_{X}(\phi) b \not p\right\}$ and let $\theta_{2}=\left\{\phi: a c_{X}(\phi) \nsubseteq \subseteq p\right\}$. I claim both $\theta_{1}$ and $\theta_{2}$ are closed under disjunction. To see this for $\theta_{1}$, suppose $\phi_{1}$ and $\phi_{2}$ are in $\theta_{1}$. Then $c_{X}\left(\phi_{1}\right) b \nsubseteq p$ and $c_{X}\left(\phi_{2}\right) b \not \subset p$. So there are $\beta_{1}, \beta_{2}, \psi_{1}$, and $\psi_{2}$ so that both for $i=1$ and $i=2$, $\phi_{i} \succ \beta_{i} \rightarrow \psi_{i}, \beta_{i} \in b$, and $\psi_{i} \notin p$. By fiddling, it follows from this that $\phi_{1} \vee \phi_{2} \succ$ $\left(\beta_{1} \wedge \beta_{2}\right) \rightarrow\left(\psi_{1} \vee \psi_{2}\right)$. But since $b$ is a theory, $\beta_{1} \wedge \beta_{2} \in b$, and since $p$ is prime, $\psi_{1} \vee \psi_{2} \notin p$. Thus $c_{X}\left(\phi_{1} \vee \phi_{2}\right) b \nsubseteq p$, so $\phi_{1} \vee \phi_{2} \in \theta_{1}$.

On the other hand, suppose $\phi_{1}$ and $\phi_{2}$ are in $\theta_{2}$. Then $a c_{X}\left(\phi_{1}\right) \nsubseteq p$ and $a c_{X}\left(\phi_{2}\right) \nsubseteq$ $p$. So there are $\alpha_{1}, \alpha_{2}, \psi_{1}$, and $\psi_{2}$ so both for $i=1$ and $i=2, \phi_{i} \succ \alpha_{i}, \alpha_{i} \rightarrow \psi_{i} \in$ $a$, and $\psi_{i} \notin p$. By further fiddling, it follows from this that $\phi_{1} \vee \phi_{2} \succ \alpha_{1} \vee \alpha_{2}$ and $\left(\alpha_{1} \vee \alpha_{2}\right) \rightarrow\left(\psi_{1} \vee \psi_{2}\right) \in a$ and $\psi_{1} \vee \psi_{2} \notin p$. So $\phi_{1} \vee \phi_{2} \in \theta_{2}$.

Since $a b \subseteq p$, it's also clear that $a \cap \theta_{1}=\emptyset$ and $b \cap \theta_{2}=\emptyset$. So by the Lindenbaum Lemma, there are prime $X$-theories $q_{1} \supseteq a$ with $q_{1} \cap \theta_{1}=\emptyset$ and $q_{2} \supseteq b$ with $q_{2} \cap \theta_{2}=$ $\emptyset$. It follows that $q_{1}$ and $q_{2}$ have the features we require.

Theorem 5. $\left\langle T_{X},{ }_{X}, \mathrm{RWQ}_{X}, \subseteq, \Pi_{X}, *_{X}, X, I_{X}\right\rangle$ is a $z$-model for each finite set of variables $X$.

Proof. Immediate from the definitions and Lemmas 33-35.

Lemma 36. $c_{X}^{a, b}$ is a closure operator; for all $t, c_{X}^{a, b}(t)$ is symmetric in a and $b$; for all prime $X$-theories $p, c_{X}^{a, b}(p)$ is also prime, and if t is already symmetric in a and $b$, then $c_{X}^{a, b}(t)=t$.

Proof. Essentially immediate from the definitions.

Lemma 37. $c_{X}^{a, b}\left(\left(c_{X}^{a, b}(t)\right)^{*} X\right)=\left(c_{X}^{a, b}(t)\right)^{*} X$.

Proof. It suffices to show that $c_{X}^{a, b}\left(\left(c_{X}^{a, b}(t)\right)^{*}\right) \subseteq\left(c_{X}^{a, b}(t)\right)^{* X}$, the converse containment being a corollary of the previous lemma.

Since $\left(c_{X}^{a, b}(t)\right)^{*}$ is obviously an $X$-theory all we need to show is that it's an $X^{a, b}$-theory. So let $\phi(a) \in\left(c_{X}^{a, b}(t)\right)^{*}$. Then $\neg \phi(a) \notin c_{X}^{a, b}(t)$. Thus since $c_{X}^{a, b}(t)$ is an $X^{a, b}$-theory, $\neg \phi(b) \notin c_{X}^{a, b}(t)$. So $\phi(b) \in\left(c_{X}^{a, b}(t)\right)^{*} X$.

Lemma 38. $c_{X}^{a, b}(t u) \subseteq c_{X}^{a, b}(t) c_{X}^{a, b}(u)$.

Proof. Let $\phi \in c_{X}^{a, b}(t u)$. Then $t u \succ^{a, b} \phi$. Thus, for some $\psi \in t u, \psi \succ^{a, b} \phi$. So there is a $\rho \in u \subseteq c_{X}^{a, b}(u)$ with $\rho \rightarrow \psi \in t \subseteq c_{X}^{a, b}(t)$. Thus $\psi \in c_{X}^{a, b}(t) c_{X}^{a, b}(u)$. So since $c_{X}^{a, b}(t) c_{X}^{a, b}(u)$ is an $X^{a, b}$-theory and $\psi \succ^{a, b} \phi$, it follows that $\phi \in c_{X}^{a, b}(t) c_{X}^{a, b}(u)$ as required.

Lemma 39. $c_{X}^{a, b}(t) c_{X}^{a, b}(u) \subseteq c_{X}^{a, b}(t) u$.

Proof. Let $\phi \in c_{X}^{a, b}(t) c_{X}^{a, b}(t)$. Then there is a $\psi \in c_{X}^{a, b}(u)$ with $\psi \rightarrow \phi \in c_{X}^{a, b}(t)$. Since $\psi \in c_{X}^{a, b}(u)$, there is a $\rho \in u$ with $\rho \succ^{a, b} \psi$. Thus by Lemma 31, RWQ $\succ^{a, b} \rho \rightarrow \psi$. So since $t \succ^{a, b} \psi \rightarrow \phi$, it follows by Lemma 11 that $t \succ^{a, b} \rho \rightarrow \phi$. Thus $\rho \rightarrow \phi \in c_{X}^{a, b}(t)$. So $\phi \in c_{X}^{a, b}(t) u$. 
Lemma 40. If $c_{X}^{a, b}(s) \subseteq p \in \Pi_{X}$, then there is a $t$ with $c_{X}^{a, b}(s) \leq c_{X}^{a, b}(t) \leq p$ and $c_{X}^{a, b}(t) \in \Pi_{X}$.

Proof. This is a straightforward application of the Lindenbaum Lemma for $X^{a, b}$ theories, so left to the reader.

Definition 12. For finite sets of variables $X \subseteq Y$, we let $t \Downarrow_{X}^{Y}=t \cap \mathscr{L}_{X}$ and $t \Uparrow_{X}^{Y}=$ $c_{Y}(t)$

Lemma 41. $\Downarrow$ and $\Uparrow$ are monotonic; $t \Downarrow_{Y}^{Z} \Downarrow_{X}^{Y}=t \Downarrow_{X}^{Z} ; t \Uparrow_{Y}^{Z} \Uparrow_{X}^{Y}=t \Uparrow_{X}^{Z}$; $\mathrm{RWQ}_{X} \Uparrow_{X}^{Y}=$ $\mathrm{RWQ}_{Y} ; t \Downarrow_{X}^{Y} \Uparrow_{X}^{Y} \subseteq t ;$ and $t \Uparrow_{X}^{Y} \Downarrow_{X}^{Y}=t$

Proof. Essentially immediate from definitions.

Lemma 42. $\left(t^{*_{Y}}\right) \Downarrow_{X}^{Y}=\left(t \Downarrow_{X}^{Y}\right)^{*_{X}}$.

Proof. $\phi \in\left(t^{*}\right) \Downarrow_{X}^{Y}$ iff $\phi \in t^{*_{Y}} \cap \mathscr{L}_{X}$ iff $\phi \in \mathscr{L}_{X}$ and $\neg \phi \notin t$ iff $\neg \phi \notin t \cap \mathscr{L}_{X}$ iff $\phi \in\left(t \Downarrow \Downarrow_{X}^{Y}\right)^{*} X$.

Lemma 43. $\Pi_{Y} \Downarrow_{X}^{Y}=\Pi_{X}$.

Proof. We leave showing $\Pi_{Y} \Downarrow_{X}^{Y} \subseteq \Pi_{X}$ to the reader. For the converse, let $p \in \Pi_{X}$ and let $\theta=\mathscr{L}_{X}-p$. Since $p$ is prime, $\theta$ is closed under disjunctions and clearly $c_{Y}(p)$ is a $Y$-theory that does not intersect $\theta$. Thus, by the Lindenbaum Lemma applied to $Y$-theories, there is a prime $Y$-theory $q$ with $p \subseteq c_{Y}(p) \subseteq q$ and $q \cap \theta=\emptyset$. It follows that $q \Downarrow_{X}^{Y}=p$. So $\Pi_{Y} \Downarrow_{X}^{Y} \supseteq \Pi_{X}$.

Lemma 44. $\left(t^{*} X\right) \Uparrow_{X}^{Y}=\left(t \Uparrow_{X}^{Y}\right)^{*_{Y}}$ and $(t s) \Uparrow_{X}^{Y}=t \Uparrow_{X}^{Y} s \Uparrow_{X}^{Y}$.

Proof. For the first of these, let $\phi \in\left(t^{*}\right) \Uparrow_{X}^{Y}$. Then $\tau_{X} \succ \phi$ for some $\tau_{X} \in t^{* X}$. Since $\tau_{X} \in t^{* X}, \neg \tau \notin t$. Thus $t \ngtr \neg \tau$. So $\neg \tau \notin t \Uparrow_{X}^{Y}$. Thus $\tau \in\left(t \Uparrow_{X}^{Y}\right)^{* Y}$, so since $\left(t \Uparrow_{X}^{Y}\right)^{*_{Y}}$ is a theory, $\phi \in\left(t \Uparrow_{X}^{Y}\right)^{*}$ as well.

For the second, first note that since $t \subseteq t \Uparrow_{X}^{Y}$ and $s \subseteq s \Uparrow_{X}^{Y}$, the left-to-right containment is obvious. For the other direction, let $\phi \in t \Uparrow_{X}^{Y} s \Uparrow_{X}^{Y}$. Then there is $\psi \in s \Uparrow_{X}^{Y}=c_{Y}(s)$ with $\psi \rightarrow \phi \in t \Uparrow_{X}^{Y}=c_{Y}(t)$. Since $\psi \in c_{Y}(s)$, there is $\sigma \in s$ with $\sigma \succ \psi$. Thus by the Deduction Lemma, $\sigma \rightarrow \psi \in \mathrm{RWQ}$. Thus, since $\psi \rightarrow \phi \in t$, $\sigma \rightarrow \phi \in t$ as well. So there is a $\tau \in t$ with $\tau \succ \sigma \rightarrow \phi$ and thus by the Deduction Lemma, $\tau \rightarrow(\sigma \rightarrow \phi) \in$ RWQ. Let $Y-X=\left\{y_{1}, \ldots, y_{n}\right\}$. Then by R3, $\forall y_{1} \ldots \forall y_{n}(\tau \rightarrow(\sigma \rightarrow \phi)) \in \mathrm{RWQ}$. So since $\tau \in t \subseteq \mathscr{L}_{X}$ and $\sigma \in s \subseteq \mathscr{L}_{X}$, A11 and a bit of fiddling give that $\tau \rightarrow\left(\sigma \rightarrow \forall y_{1} \ldots \forall y_{n} \phi\right) \in$ RWQ. Thus since $\tau \in t$, $\sigma \rightarrow \forall y_{1} \ldots \forall y_{n} \phi \in t$. So since $\sigma \in s, \forall y_{1} \ldots \forall y_{n} \phi \in t s$. Thus $\phi \in(t s) \Uparrow_{X}^{Y}$.

Lemma 45. $\left(t u \Uparrow_{X}^{Y}\right) \Downarrow_{X}^{Y} \subseteq t \Downarrow_{X}^{Y} u$.

Proof. Let $\phi \in\left(t u \Uparrow_{X}^{Y}\right) \Downarrow_{X}^{Y}$. Then $\phi \in \mathscr{L}_{X}$ and there is a $\psi \in u \Uparrow_{X}^{Y}$ with $\psi \rightarrow \phi \in t$. Since $\psi \in u \Uparrow_{X}^{Y}$, for some $\rho \in u, \rho \succ \psi$. Thus $\rho \rightarrow \psi \in \mathrm{RWQ}$. Let $Y-X=$ $\left\{y_{1}, \ldots, y_{n}\right\}$. Then using R3 and A11, we see that $\rho \rightarrow \forall y_{1} \ldots \forall y_{n} \psi \in$ RWQ. It follows that $\forall y_{1} \ldots \forall y_{n} \psi \in u$. But repeated applications of A10 and R1 give that $\forall y_{1} \ldots \forall y_{n} \psi \rightarrow \psi \in$ RWQ. So since $\psi \rightarrow \phi \in t, \forall y_{1} \rightarrow \forall y_{n} \psi \rightarrow \phi \in t$ as well. But also $\forall y_{1} \rightarrow \forall y_{n} \psi \rightarrow \phi \in \mathscr{L}_{X}$. So $\forall y_{1} \rightarrow \forall y_{n} \psi \rightarrow \phi \in t \Downarrow_{X}^{Y}$, so $\phi \in t \Downarrow_{X}^{Y} u$. 
Lemma 46. $I_{Y}(t)(P) \cap \operatorname{Dom}_{X}^{i}=I_{X}\left(t \Downarrow_{X}^{Y}\right)(P)$.

Proof. Immediate from definitions.

Lemma 47. If $t \Downarrow_{X}^{Y} \subseteq a$, then for some $p \in \Pi_{Y}, t \subseteq p$ and $t \Downarrow_{X}^{Y}=a$.

Proof. Let $s=c_{Y}(t \cup a)$ and let $\theta=\mathscr{L}_{X}-a$ Clearly $s$ is a $Y$-theory. I claim $s \cap \theta=\emptyset$.

To see this, suppose $\phi \in s \cap \theta$. Then $t \cup a \succ \phi$. So there is $\tau \in t$ and $\alpha \in a$ so that $\tau \wedge \alpha \succ \phi$. Thus by the Deduction Lemma, $(\tau \wedge \alpha) \rightarrow \phi \in$ RWQ. Let $Y-X=\left\{y_{1}, \ldots, y_{n}\right\}$. By fiddling, it's clear then that $\left(\forall y_{1} \ldots \forall y_{n} \tau \wedge \forall y_{1} \ldots \forall y_{n} \alpha\right) \rightarrow$ $\phi \in$ RWQ as well. But $\forall y_{1} \ldots \forall y_{n} \tau \in t \Downarrow_{X}^{Y} \subseteq a$ and $\forall y_{1} \ldots \forall y_{n} \alpha \in a$. So $\forall y_{1} \ldots \forall y_{n} \tau \wedge$ $\forall y_{1} \ldots \forall y_{n} \alpha \in a$, from which it follows that $\phi \in a$, which is impossible. So $s \cap \theta=\emptyset$. Thus the Lindenbaum Lemma applied to $Y$-theories gives that there is a $p \in \Pi_{Y}$ with $s \subseteq p$ and $p \cap \theta=\emptyset$. Since $a \subseteq s$ and $t \subseteq s, a \subseteq p$ and $t \subseteq p$. And since $p \cap \theta=\emptyset$, $p \subseteq a$, showing that $p$ has the required features.

Lemma 48. If $a$ and $b$ are in $X$, then $c_{Y}^{a, b}\left(t \Uparrow_{X}^{Y}\right)=c_{X}^{a, b}(t) \Uparrow_{X}^{Y}$.

Proof. If $\phi \in c_{Y}^{a, b}\left(t \Uparrow_{X}^{Y}\right)$, then $\phi \in \mathscr{L}_{Y}$ and $t \Uparrow_{X}^{Y} \succ^{a, b} \phi$. Thus, $\tau_{Y} \succ^{a, b} \phi$ for some $\tau_{Y} \in$ $t \Uparrow_{X}^{Y}$. So by Lemma 31, RWQ $\succ^{a, b} \tau_{Y} \rightarrow \phi$. Since $\tau_{Y} \in t \Uparrow_{X}^{Y}, \tau_{X} \succ \tau_{Y}$ for some $\tau_{X} \in t$. So RWQ $\succ \tau_{X} \rightarrow \tau_{Y}$. Thus, by fiddling, RWQ $\succ^{a, b} \tau_{X} \rightarrow \phi$. Let $Y-X=\left\{y_{1}, \ldots, y_{n}\right\}$. Then by repeated application of R3, RWQ $\succ^{a, b} \forall y_{1} \ldots \forall y_{n}\left(\tau_{X} \rightarrow \phi\right)$. So by fiddling, RWQ $\succ \tau_{X} \rightarrow \forall y_{1} \ldots \forall y_{n} \phi$. It follows that $\forall y_{1} \ldots \forall y_{n} \phi \in c_{X}^{a, b}(t)$. So $\phi \in c_{X}^{a, b}(t) \Uparrow_{X}^{Y}$. Thus $c_{Y}^{a, b}\left(t \Uparrow_{X}^{Y}\right) \subseteq c_{X}^{a, b}(t) \Uparrow_{X}^{Y}$.

Now let $\phi \in c_{X}^{a, b}(t) \Uparrow_{X}^{Y}$. Then $\tau \succ \phi$ for some $\tau \in c_{X}^{a, b}(t)$. Since $\tau \in c_{X}^{a, b}(t), \tau^{\prime} \succ^{a, b} \tau$ for some $\tau^{\prime} \in t$. Since $\tau^{\prime} \in t, \tau^{\prime} \in t \Uparrow_{X}^{Y}$. So since $\tau^{\prime} \succ^{a, b} \tau, \tau \in c_{Y}^{a, b}\left(t \Uparrow_{X}^{Y}\right)$. Finally, since $\tau \succ \phi$, this gives that $\phi \in c_{Y}^{a, b}\left(t \Uparrow_{X}^{Y}\right)$. So $c_{Y}^{a, b}\left(t \Uparrow_{X}^{Y}\right) \supseteq c_{X}^{a, b}(t) \Uparrow_{X}^{Y}$, completing the proof.

Lemma 49. If $a \in Y-X$ and $b \in X$, then $c_{Y}^{b, a}\left(t \Uparrow_{X}^{Y}\right) \Downarrow_{X}^{Y} \subseteq t$

Proof. Let $\phi \in c_{Y}^{b, a}\left(t \Uparrow_{X}^{Y}\right) \Downarrow_{X}^{Y}$. Then $\phi \in \mathscr{L}_{X}$ and $\phi \in c_{Y}^{b, a}\left(t \Uparrow_{X}^{Y}\right)$. So $t \Uparrow_{X}^{Y} \succ^{b, a} \phi$, and thus $\tau_{Y} \succ^{b, a} \phi$ for some $\tau_{y} \in t \Uparrow_{X}^{Y}$. And since $\tau_{Y} \in t \Uparrow_{X}^{Y}, \tau_{X} \succ \tau_{Y}$ for some $\tau_{X} \in t$. It follows that $\tau_{X} \succ^{b, a} \phi$. Thus by Lemma 31, $\tau_{X}(a / / b) \rightarrow \phi(a / / b) \in$ RWQ. But since $a \in Y-X$ and $\tau_{X}$ and $\phi$ are in $\mathscr{L}_{X}, a$ does not occur in $\tau_{X}$ or in $\phi$. So $\tau_{X}(a / / b)=\tau$ and $\phi(a / / b)=\phi$. So in fact $\tau_{X} \rightarrow \phi \in \mathrm{RWQ}$. Thus since $t$ is a theory and $\tau_{X} \in t$, $\phi \in t$ as well, as required.

Lemma 50. for all $V \supseteq X, t \Uparrow_{X}^{V} \Downarrow_{W}^{V}=t \Downarrow_{Y}^{X} \Uparrow_{Y}^{W}$, as long as $X \cap W=Y$

Proof. Let $Y \cap Z=X, W \supseteq Y$ and let $\phi \in t \Downarrow_{X}^{Y} \Uparrow_{X}^{Z}$. Then $\tau_{X} \rightarrow \phi \in$ RWQ for some $\tau_{X} \in t \Downarrow_{X}^{Y}=t \cap \mathscr{L}_{X}$. Since $\tau_{X} \in t, \tau_{X} \in t \Uparrow_{Y}^{W}$. But then since $\mathscr{L}_{X} \subseteq \mathscr{L}_{Z}$, we also have that $\tau_{X} \in \Uparrow_{Y}^{W} \Downarrow_{Z}^{W}$. So $t \Downarrow_{X}^{Y} \Uparrow_{X}^{Z} \subseteq t \Uparrow_{Y}^{W} \Downarrow_{Z}^{W}$.

Now let $\phi \in t \Uparrow_{Y}^{W} \Downarrow_{Z}^{W}=t \Uparrow_{Y}^{W} \cap \mathscr{L}_{Z}$. Then $\tau_{Y} \rightarrow \phi \in$ RWQ for some $\tau_{Y} \in t$. Let $Z-Y=\left\{z_{1}, \ldots, z_{n}\right\}$. Then by repeated application of R3, $\forall z_{1} \ldots \forall z_{n}\left(\tau_{Y} \rightarrow \phi\right) \in$ RWQ as well. So by fiddling, $\tau_{Y} \rightarrow \forall z_{1} \ldots \forall z_{n} \phi \in$ RWQ. So since $\tau_{Y} \in t$, clearly $\forall z_{1} \ldots \forall z_{n} \phi \in t$. But clearly $\forall z_{1} \ldots \forall z_{n} \phi \in \mathscr{L}_{Z \cap Y}=\mathscr{L}_{X}$. So in fact $\forall z_{1} \ldots \forall z_{n} \phi \in t \Downarrow \Downarrow_{X}^{Y}$, from which it follows that $\phi \in t \Downarrow_{X}^{Y} \Uparrow_{X}^{Z}$. 
Theorem 6. The data $\mathbf{M}, c b, \Downarrow$, define a stratified model.

Proof. Immediate from the above.

Lemma 51. If $\phi \in \mathscr{L}_{X}$, then $\mathbf{M}, X, t \vDash \phi$ iff $\phi \in t$.

Proof. By induction on $\phi$. The cases for atoms and conjunctions are trivial. For the conditional case, first suppose that $\phi \rightarrow \psi \in t$. Let $\mathbf{M}, X, u \vDash \phi$. Then by IH, $\phi \in u$. So

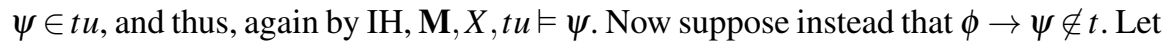

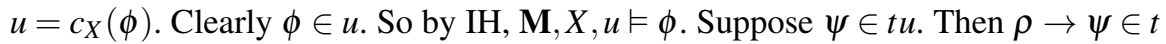
for some $\rho \in u$. But if $\rho \in u$, then $\phi \rightarrow \rho \in \mathrm{RWQ}$, and hence $\phi \rightarrow \psi \in t$, which is a contradiction. So $\psi \notin t u$. Thus by IH, M, $X, t u \not \forall \psi$. So $\mathbf{M}, X, t \not \forall \phi \rightarrow \psi$.

For the universal case, first suppose $M, X, t \vDash \forall k \phi(k)$. Then there are $Y \supsetneq X$ and $y \in Y-X$ so that $M, Y, t \Uparrow_{X}^{Y} \vDash \phi(y)$. So by IH, $\phi(y) \in t \Uparrow_{X}^{Y}=c_{Y}(t)$. So $t \vDash \phi(y)$. So for some $\tau \in t, \tau \rightarrow \phi(y) \in \mathrm{RWQ}$. Since $\tau \in t \subseteq \mathscr{L}_{X}$ and $y \in Y-X$, it follows by some fiddling that $\tau \rightarrow \forall k \phi(k) \in \mathrm{RWQ}$ as well. So since $\tau \in t, \forall k \phi(k) \in t$ as well.

Finally, suppose $\forall k \phi(k) \in t$. Let $Y \supsetneq X$ and $y \in Y$. Then $\phi(y) \in c_{Y}(t)=t \Uparrow_{X}^{Y}$. So by $\mathrm{IH}, \mathbf{M}, Y, t \Uparrow_{X}^{Y} \vDash \phi(y)$. It follows that $\mathbf{M}, X, t \vDash \forall k \phi(k)$ as required.

Theorem 7. If $\operatorname{Str} \vDash \phi$, then $\phi \in \mathrm{RWQ}$.

Proof. Let $\phi \in \mathscr{L}_{X}$ and suppose $\phi \notin \mathrm{RWQ}$. Then $\phi \notin \mathrm{RWQ}_{X}$. So by Lemma 51, $\mathbf{M}, X, \mathrm{RWQ}_{X} \not \models \phi$. Thus $\mathbf{S t r} \not \models \phi$.

\section{References}

1. A. R. Anderson and N. D. Belnap. Entailment: The Logic of Relevance and Necessity, Vol. I. Princeton University Press, 1975.

2. A. R. Anderson, N. D. Belnap, and J. M. Dunn. Entailment: The Logic of Relevance and Necessity, Vol. II. Princeton University Press, 1992.

3. R. T. Brady. Natural deduction systems for some quantified relevant logics. Logique Et Analyse, 27(108):355-378, 1984.

4. R. T. Brady. Some concerns regarding ternary-relation semantics and truth-theoretic semantics in general. IfCoLog Journal of Logics and their Applications, 4(3):755-781, 2017.

5. J. M. Dunn. Relevance logic and entailment. In Handbook of philosophical logic, pages 117-224. Springer, 1986.

6. J. M. Dunn. Star and perp: Two treatments of negation. Philosophical Perspectives, 7:331357, 1993.

7. K. Fine. Models for entailment. Journal of Philosophical Logic, 3(4):347-372, 1974.

8. K. Fine. Reasoning with arbitrary objects. Blackwell, 1985.

9. K. Fine. Semantics for quantified relevance logic. Journal of Philosophical Logic, 17(1):2759, 1988

10. K. Fine. Incompleteness for quantified relevance logics, pages 205-225. Directions in Relevant Logic. Springer, 1989.

11. K. Fine and N. Tennant. A defence of arbitrary objects. Proceedings of the Aristotelian Society, Supplementary Volumes, 57:55-89, 1983.

12. N. Galatos, P. Jipsen, T. Kowalski, and H. Ono. Residuated Lattices: An Algebraic Glimpse at Substructural Logics. Elsevier, 2007.

13. R. I. Goldblatt. Topoi: The Catergorical Analysis of Logic. Dover Publications, 2006. 
14. F. W. Lawvere. Adjointness in foundations. Dialectica, 23(34):281-296, 1969.

15. S. A. Logan. Notes on stratified semantics. Journal of Philosophical Logic, 48(4):749-786, 2019.

16. E. D. Mares. Semantics for relevance logic with identity. Studia Logica, 51(1):1-20, 1992.

17. E. D. Mares. Relevant Logic: A Philosophical Interpretation. Cambridge Univeristy Press, 2004.

18. E. D. Mares and R. Goldblatt. An alternative semantics for quantified relevant logic. Journal of Symbolic Logic, 71(1):163-187, 2006.

19. Y. Maruyama. Full lambek hyperdoctrine: categorical semantics for first-order substructural logics. In International Workshop on Logic, Language, Information, and Computation, pages 211-225. Springer, 2013.

20. A. M. Pitts. Categorical logic. Technical report, University of Cambridge, Computer Laboratory, 1995.

21. S. Read. Relevant Logic: A Philosophical Examination of Inference. Blackwell, 1988.

22. G. Restall. Negation in relevant logics (how I stopped worrying and learned to love the Routley star). In D. M. Gabbay and H. Wansing, editors, What is Negation?, pages 53-76. Kluwer Academic Publishers, 1999.

23. R. Routley and V. Routley. The semantics of first degree entailment. Noús, pages 335-359, 1972.

24. R. A. Seely. Hyperdoctrines, natural deduction and the Beck condition. Mathematical Logic Quarterly, 29(10):505-542, 1983.

25. J. Slaney. A general logic. Australasian Journal of Philosophy, 68(1):74-88, 1990. 